\title{
Scattering polarization in the presence of magnetic and electric fields
}

\author{
Yee Yee Oo ${ }^{a}$, M. Sampoorna ${ }^{\text {b,d }}$, K.N. Nagendra ${ }^{b}$, \\ Sharath Ananthamurthy ${ }^{\mathrm{c}}$, G. Ramachandran ${ }^{\mathrm{b}, *}$ \\ ${ }^{a}$ Department of Physics, Mandalay University, Mandalay, Myanmar \\ ${ }^{\mathrm{b}}$ Indian Institute of Astrophysics, Bangalore 560 034, India \\ ${ }^{\mathrm{c}}$ Department of Physics, Bangalore University, Bangalore 560 056, India \\ d Joint Astronomy Program, Department of Physics, IISc, Bangalore 560 012, India
}

Received 6 February 2007; received in revised form 24 April 2007; accepted 29 April 2007

\begin{abstract}
The polarization of radiation by scattering on an atom embedded in combined external quadrupole electric and uniform magnetic fields is studied theoretically. Limiting cases of scattering under Zeeman effect, and Hanle effect in weak magnetic fields are discussed. The theory is general enough to handle scattering in intermediate magnetic fields (Hanle-Zeeman effect) and for arbitrary orientation of magnetic field. The quadrupolar electric field produces asymmetric line shifts, and causes interesting level-crossing phenomena either in the absence of an ambient magnetic field, or in its presence. It is shown that the quadrupolar electric field produces an additional depolarization in the $Q / I$ profiles and rotation of the plane of polarization in the $U / I$ profile over and above that arising from magnetic field itself. This characteristic may have a diagnostic potential to detect steady-state and time-varying electric fields that surround radiating atoms in solar atmospheric layers.
\end{abstract}

(C) 2007 Elsevier Ltd. All rights reserved.

Keywords: Atomic processes; Polarization; Scattering; Magnetic field; Line profiles

\section{Introduction}

Scattering of polarized radiation by an atom is a topic of considerable interest to astrophysics ever since Hale [1] first observed polarization related to Zeeman effect in spectral lines originating in Sun spots. The polarized radiation is usually expressed in terms of the Stokes parameters. The concept of scattering matrix connecting the Stokes vector $\mathbf{S}^{\prime}$ of incident radiation to the Stokes vector $\mathbf{S}$ of scattered radiation was introduced quite early in the context of Rayleigh scattering [2]. Polarized radiation in spectral lines formed in the presence of an external magnetic field has been studied widely and a comprehensive theoretical framework has been developed [3-15]. The Hanle effect is a depolarizing phenomenon which arises due to "partially

\footnotetext{
*Corresponding author. Tel.: + 918025530672 ; fax: +918025534043.

E-mail address: gr@iiap.res.in (G. Ramachandran).
} 
overlapping' magnetic substates in the presence of weak magnetic fields, when the splitting produced is of the same order or less than the natural widths. Favati et al. [16] proposed the name 'second Hanle effect' for a similar effect in 'electrostatic fields'. Casini and Landi Degl'Innocenti [17] have discussed the problem in the presence of electric and magnetic fields for the particular case of hydrogen Lyman $\alpha$ line. It was followed by a more recent paper by Casini [18]. The relative contributions of static external electric fields, motional electric fields and magnetic fields in the case of hydrogen Balmer lines, have been studied by Brillant et al. [19]. A historical perspective and extensive references to earlier literature on polarized line scattering can be found in Stenflo [12], Trujillo Bueno et al. [20] and Landi Degl'Innocenti and Landolfi [21].

A quantum electrodynamic theory of Hanle-Zeeman redistribution matrices has been developed by Bommier [10,11] and Landi Degl'Innocenti and co-workers (see the book by [21]). The formulation presented in $[10,11]$ includes the effects of partial frequency redistribution (PRD) in line scattering for a two-level atom. It is a perturbation theory, in which PRD effects appear in the fourth order. The theory presented in [21] and references cited therein, considers only complete frequency redistribution (CRD) in line scattering.

A classical theory of line scattering PRD for the Hanle-Zeeman effect has been formulated by Bommier and Stenflo [15]. This theory is non-perturbative and describes the scattering process in a transparent way. The classical theory for Hanle-Zeeman scattering developed by Stenflo [14] considered only coherent scattering in the laboratory frame. In [15] the redistribution matrices were derived in the atomic rest frame. The corresponding laboratory frame redistribution matrices have been derived in [22]. The equivalence between the classical (non-perturbative theory) and quantum electrodynamic (perturbative theory) redistribution matrices for the triplet case is established in [23]. In all these papers only the dipole type line scattering transitions in the presence of pure magnetic fields is considered. Taking into account all higher order multipoles as well, polarization of line radiation in the presence of external electric quadrupole and uniform magnetic fields was studied [24,25], where scattering of radiation by atoms, however, was not considered.

The purpose of the present paper is to develop a quantum electrodynamical approach to scattering processes in the presence of external electric and magnetic fields of 'arbitrary strengths', taking also into consideration all other multipole type transitions apart from the usually dominant electric dipole transition. The atomic electron is represented using non-relativistic quantum theory including spin. The radiation field is described in terms of its electric and magnetic multipole states, in a second quantized formalism. The external electric field is assumed to be 'quadrupolar' in nature, while the magnetic field is uniform and arbitrarily oriented with reference to the principal axes frame (PAF) of the electric quadrupole field. This general formalism can be employed also to solve the scattering problems involving linear steady-state electric fields at the radiating atom.

In Section 2 we describe the theoretical formulation. In Section 3 the scattering matrix for the general physical situation is derived. The particular case of the dipole transitions for a triplet is also considered, for the purpose of comparison with Stenflo [14] in the pure magnetic field limit. Section 4 contains numerical results and discussions. Conclusions are presented in Section 5.

\section{Theoretical formalism for scattering}

We consider polarized radiation incident on an atom along an arbitrary direction $\left(\theta^{\prime}, \phi^{\prime}\right)$ and getting scattered into a direction $(\theta, \phi)$ with respect to a conveniently chosen right-handed Cartesian coordinate system, referred to as the astrophysical reference frame (ARF) and shown as $(X, Y, Z)$ in Fig. 1 . If $v^{\prime}$ and $v$ denote, respectively, the frequencies of the incident and scattered radiation, we may define wave vectors $\mathbf{k}^{\prime}$ and $\mathbf{k}$ with polar co-ordinates $\left(k^{\prime}, \theta^{\prime}, \phi^{\prime}\right)$ and $(k, \theta, \phi)$ where $k^{\prime}=2 \pi v^{\prime}=\omega^{\prime}$ and $k=2 \pi v=\omega$ in natural units with $\hbar=1, c=1$ and mass of the electron $m_{e}=1$. The atom is exposed to an external magnetic field $\mathbf{B}$ with strength $B$, directed along $\left(\theta_{B}, \phi_{B}\right)$ and an electric quadrupole field characterized by strength $A$ and asymmetry parameter $\eta$ in its PAF, which is denoted by $\left(X_{Q}, Y_{Q}, Z_{Q}\right)$ in Fig. 1. The transformation to PAF from ARF is achieved by a rotation $R\left(\alpha_{Q}, \beta_{Q}, \gamma_{Q}\right)$ through Euler angles $\left(\alpha_{Q}, \beta_{Q}, \gamma_{Q}\right)$ as defined by Rose [26]. The magnetic field $\mathbf{B}$ is directed along $\left(\widetilde{\theta}_{B}, \widetilde{\phi}_{B}\right)$ with respect to PAF. Following Rose [26], we define left and right circular states of polarization $\hat{\varepsilon}_{\mu}(\mu= \pm 1)$, respectively, which are mutually orthogonal to each other and to $\mathbf{k}$. We use here the symbol $\hat{\varepsilon}_{\mu= \pm 1}$ instead of $\hat{\mathbf{u}}_{p= \pm 1}$ employed in Rose [26]. Likewise $\hat{\varepsilon}_{\mu^{\prime}= \pm 1}^{\prime}$, which are orthogonal to 


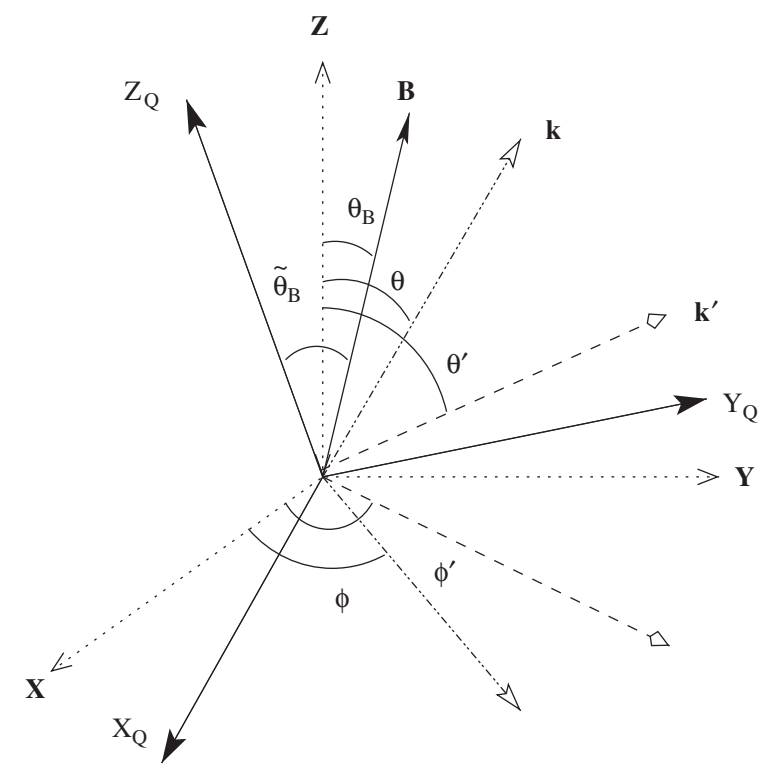

Fig. 1. The scattering geometry: $\left(X_{Q}, Y_{Q}, Z_{Q}\right)$ refers to the principal axes frame (PAF) characterizing the electric quadrupole field. The radiation is incident along $\left(\theta^{\prime}, \phi^{\prime}\right)$ and scattered along $(\theta, \phi)$ with respect to the astrophysical reference frame (ARF) denoted by $(X, Y, Z)$. The magnetic field $\vec{B}$ is oriented along $\left(\widetilde{\theta}_{B}, \widetilde{\phi}_{B}\right)$ with reference to PAF and $\left(\theta_{B}, \phi_{B}\right)$ with reference to the ARF (the azimuthal angles $\widetilde{\phi}_{B}$ and $\phi_{B}$ are not marked in the figure).

$\mathbf{k}^{\prime}$. Any arbitrary state of polarization $\hat{\varepsilon}^{\prime}$ of the incident radiation may then be expressed as $\hat{\varepsilon}^{\prime}=$ $c_{+1}^{\prime} \hat{\varepsilon}_{+1}^{\prime}+c_{-1}^{\prime} \hat{\varepsilon}_{-1}^{\prime}$ using appropriate coefficients $c_{ \pm 1}^{\prime}$, which are in general complex and satisfy $\left|c_{+1}^{\prime}\right|^{2}+\left|c_{-1}^{\prime}\right|^{2}=1$.We, therefore, denote the orthonormal states of polarized incident radiation by $\left|\mathbf{k}^{\prime}, \mu^{\prime}\right\rangle$, with $\mu^{\prime}= \pm 1$. We seek the probability for scattering into two polarized states of scattered radiation $|\mathbf{k}, \mu\rangle$, $\mu= \pm 1$ on an atom which is initially in a state $\psi_{i}$ with energy $E_{i}$ before scattering and makes a transition to a final state $\psi_{f}$ with energy $E_{f}$, in the process of scattering of polarized radiation.

\subsection{Energy levels of an atom in electric quadrupole and uniform magnetic fields}

The energy levels of an electron in an atom are primarily determined by the Hamiltonian

$$
H_{0}=-\frac{1}{2} \nabla^{2}+V(r)
$$

where $V(r)$ denotes its Coulomb interaction with the nucleus. If we start with the Dirac equation [17] and use its non-relativistic reduction, terms like spin-orbit interaction may also be included in $H_{0}$. In the absence of external fields, the energy levels of the atom are determined by

$$
H_{0}^{A}=\sum_{i=1}^{Z} H_{0}(i)+\sum_{i>j=1}^{Z} \frac{e^{2}}{r_{i j}},
$$

where $e$ denotes the charge of the electron, $r_{i j}=\left|\mathbf{r}_{i}-\mathbf{r}_{j}\right|$ and $Z$ denotes the atomic number. If $E$ denotes an energy level and $\psi$ the corresponding wave function of the atom with total angular momentum $J$, it is well known in the context of Zeeman effect that $E$ gets split into $(2 J+1)$ equally spaced levels $E_{M}=E+g B M$ with corresponding energy eigenstates $|J M\rangle_{\mathbf{B}}, M=J, J-1, \ldots,-J+1,-J$, when the atom is exposed to an external uniform magnetic field $\mathbf{B}$ with strength $B$. The states $|J M\rangle_{\mathbf{B}}$ are defined with the axis of quantization chosen along $\mathbf{B}$ and $g$ denotes the magnetic-gyro ratio or Landé $g$-factor. For $B<100$ gauss, when $g B$ is of the same order as the width of a line, Hanle effect [27] takes place. For a line in the optical range, the region $100<B<1000$ gauss is generally referred to as the Hanle-Zeeman regime. For $B<1$ gauss, one has to pay attention to the interaction of electron with the magnetic and electric moments of the nucleus, which give rise 
to hyperfine splitting [28,29]. If the atom is exposed to an external electric quadrupole field either by itself or in combination with $\mathbf{B}$, the splitting of the energy levels is not, in general, equally spaced $[24,25,30,31]$ and in such scenarios, the atomic Hamiltonian in PAF is given by

$$
H_{A}=H_{0}^{A}+g \mathbf{J} \cdot \mathbf{B}+A\left[2 J_{z}^{2}-J_{x}^{2}-J_{y}^{2}+\eta\left(J_{x}^{2}-J_{y}^{2}\right)\right] .
$$

The split energy levels may be denoted by $E_{s}$, where $s$ takes values $s=1,2, \ldots,(2 J+1)$ starting from the lowest level $(s=1)$ for a given $J$. The corresponding energy eigenstates may be denoted by $|J, s\rangle$, which are expressible as

$$
|J, s\rangle=\sum_{M=-J}^{J} a_{M}^{s}\left(A, \eta, B, \widetilde{\theta}_{B}, \widetilde{\phi}_{B}\right)|J M\rangle_{Q}, \quad s=1,2, \ldots,(2 J+1),
$$

where $|J M\rangle_{Q}$ are defined with the quantization axis chosen along the $Z$-axis, $Z_{Q}$ of the PAF. The notation $c_{m_{u}}^{i}$ was used in [25] for $J=1,3 / 2$ to denote the expansion coefficients, without any specified convention for ordering of the levels. We may rewrite Eq. (4) as

$$
|J, s\rangle=\sum_{m=-J}^{J} c_{m}^{s}|J m\rangle,
$$

in terms of the $|J m\rangle$ states, which are defined with respect to the $Z$-axis of ARF chosen as the quantization axis. Clearly,

$$
c_{m}^{s}=\sum_{M=-J}^{J} D_{m M}^{J}\left(\alpha_{Q}, \beta_{Q}, \gamma_{Q}\right) a_{M}^{s}\left(A, \eta, B, \widetilde{\theta}_{B}, \widetilde{\phi}_{B}\right),
$$

where $D_{m M}^{J}$ denotes Wigner's $D$-functions defined in [26]. Hence $c_{m}^{s}$ depend on $\alpha_{Q}, \beta_{Q}, \gamma_{Q}, A, \eta$, B. If the magnetic field is absent, the $c_{m}^{s}$ depend only on $\alpha_{Q}, \beta_{Q}, \gamma_{Q}, A, \eta$ since $a_{M}^{s}$ in that case [24] depend only on $A$ and $\eta$. It may be noted that the frame of reference employed in [24,25] was PAF itself, i.e., $\alpha_{Q}, \beta_{Q}, \gamma_{Q}=0$; hence $\theta_{B}, \phi_{B}$ was used there instead of the $\widetilde{\theta}_{B}, \widetilde{\phi}_{B}$ here and the Euler angles $\left(\alpha_{Q}, \beta_{Q}, \gamma_{Q}\right)$ find no mention there. It may be added that $D_{m M}^{J}(0,0,0)=\delta_{m M}$. On the other hand, if the electric quadrupole field is absent and the atom is exposed only to a magnetic field $\mathbf{B}$ directed along $\left(\theta_{B}, \phi_{B}\right)$, it is clear that

$$
c_{m}^{s}=\sum_{M=-J}^{J} D_{m M}^{J}\left(\phi_{B}, \theta_{B}, 0\right) \delta_{M, s-J-1},
$$

which reduces to

$$
c_{m}^{s}=\delta_{s, J+m+1},
$$

if the field $\mathbf{B}$ is along the $Z$-axis of ARF itself.

In general, therefore, when the energy levels of an atom are defined through $H_{A} \psi_{n}=E_{n} \psi_{n}$, the atomic wave functions $\psi_{n}$ are of the form given by Eq. (5), which specialize appropriately to $|J M\rangle_{\mathbf{B}}$ or $|J m\rangle$ if Eq. (7) or Eq. (8) is used instead of Eq. (6). Thus, in general, the complete set of orthonormal energy eigenstates of an atom in a combined external electric quadrupole and uniform magnetic field environment may be denoted by $\left\{\psi_{n}\right\}$, where $n$ is used as a collective index, which includes the serial number $s_{n}$ along with the total angular momentum $J_{n}$ and all other quantum numbers which may be needed to specify each $\psi_{n}$ uniquely. In the presence of a pure magnetic field $\mathbf{B}$, the magnetic quantum number $M_{n}$ replaces $s_{n}$ through the $\delta$-function in Eq. (7). Moreover, if $\mathbf{B}$ is along the $Z$-axis of ARF itself, $s_{n}$ gets replaced by $m_{n}$ through the $\delta$-function in Eq. (8).

In general, a summation over $n$ as in $\sum_{n}\left|\psi_{n}\right\rangle\left\langle\psi_{n}\right|=1$, implies a summation with respect to $s_{n}$ as well. This summation over $s_{n}$ may be replaced by a summation with respect to $M_{n}$ or $m_{n}$ in some particular cases as mentioned above. The initial and final states of the atom before and after scattering are denoted by $\psi_{i}$ and $\psi_{f}$. They also belong to $\left\{\psi_{n}\right\}$. We use the short-hand notation

$$
|i\rangle=\left|\psi_{i} ; \mathbf{k}^{\prime}, \mu^{\prime}\right\rangle ; \quad|f\rangle=\left|\psi_{f} ; \mathbf{k}, \mu\right\rangle .
$$




\subsection{Interaction of atom with the radiation field}

It is well known that the local minimal coupling, i.e., $\bar{\psi} \gamma_{v} \psi A_{v}$ (with implied summation over $v$ ) of the Dirac field $\psi$ and the electromagnetic field represented by the four potential $A_{v}, v=1, \ldots, 4$ is the fundamental interaction responsible for all electrodynamical process involving photons and electrons [32,33]. In the interaction representation, $\psi$ and $A_{v}$ satisfy the free field equations of Dirac and Maxwell, respectively. The quantity $\bar{\psi}=\psi^{\dagger} \gamma_{4}$, where $\psi^{\dagger}$ denotes the hermitian conjugate of $\psi$ and $\gamma_{1}, \gamma_{2}, \gamma_{3}, \gamma_{4}$ are $4 \times 4$ Dirac matrices. To facilitate calculations using the atomic wave functions $\left\{\psi_{n}\right\}$, we may use the non-relativistic two componental forms of $\psi$ and $\bar{\psi}$ in $c$ number theory for electrons, retain the Maxwell field in $q$ number theory and represent the interaction of the radiation field in the Coulomb gauge with the atom as

$$
H_{\text {int }}=\mathrm{e}^{\mathrm{i} H_{A} t} \sum_{j=1}^{Z} e\left[-i \mathbf{A}\left(\mathbf{r}_{j}, t\right) \cdot \nabla_{j}+\frac{1}{2} \sigma_{j} \cdot\left(\nabla_{j} \times \mathbf{A}\left(\mathbf{r}_{j}, t\right)\right)\right] \mathrm{e}^{-\mathrm{i} H_{A} t},
$$

where $\sigma_{j}$ denote the Pauli spin matrices of the electron labeled $j$ located at $\mathbf{r}_{j}$ and $Z$ denotes the atomic number. The quantum field variable $\mathbf{A}(\mathbf{r}, t)$ in interaction representation may be expressed as

$$
\mathbf{A}\left(\mathbf{r}_{j}, t\right)=\frac{1}{(2 \pi)^{3 / 2}} \int \frac{\mathrm{d}^{3} k^{\prime \prime}}{\sqrt{2 \omega^{\prime \prime}}} \sum_{\mu^{\prime \prime}}\left[a_{\mathbf{k}^{\prime \prime} \mu^{\prime \prime}} \mathbf{A}_{\mathbf{k}^{\prime \prime} \mu^{\prime \prime}}(\mathbf{r}) \mathrm{e}^{-\mathrm{i} \omega^{\prime \prime} t}+a_{\mathbf{k}^{\prime \prime} \mu^{\prime \prime}}^{+} \mathbf{A}_{\mathbf{k}^{\prime \prime} \mu^{\prime \prime}}(\mathbf{r})^{*} \mathrm{e}^{\mathrm{i} \omega^{\prime \prime} t}\right],
$$

where $\omega^{\prime \prime}=\left|\mathbf{k}^{\prime \prime}\right|$ and the creation and annihilation operators, denoted by $a_{\mathbf{k} \mu}^{+}$and $a_{\mathbf{k} \mu}$, respectively, satisfy the commutation relation

$$
\left[a_{\mathbf{k} \mu}, a_{\mathbf{k}^{\prime} \mu^{\prime}}^{+}\right]=\delta\left(\mathbf{k}-\mathbf{k}^{\prime}\right) \delta_{\mu \mu^{\prime}}
$$

for any pair $\mathbf{k}, \mu$ and $\mathbf{k}^{\prime}, \mu^{\prime}$ in general, while

$$
\mathbf{A}_{\mathbf{k} \mu}(\mathbf{r})=\hat{\varepsilon}_{\mu} \mathrm{e}^{\mathrm{ik} \cdot \mathbf{r}}
$$

denotes a $c$ number and $\mathbf{A}_{\mathbf{k} \mu}(\mathbf{r})^{*}$ denotes its complex conjugate. In particular, the operators are also used to generate the initial and final states of radiation in Eq. (9) through

$$
\left|\mathbf{k}^{\prime} \mu^{\prime}\right\rangle=a_{\mathbf{k}^{\prime} \mu^{\prime}}^{+}|\rangle_{0} ; \quad\langle\mathbf{k} \mu|=_{0}\langle| a_{\mathbf{k} \mu},
$$

where |\rangle$_{0}$ denotes the vacuum state of the radiation field.

\subsection{The scattering process}

The $S$-matrix for scattering may be defined, as usual [32-34], by

$$
S=\lim _{\substack{t \rightarrow \infty \\ t_{0} \rightarrow-\infty}} U\left(t, t_{0}\right),
$$

where the evolution operator satisfies

$$
U\left(t, t_{0}\right)=1-i \int_{t_{0}}^{t} \mathrm{~d} t^{\prime} H_{\text {int }}\left(t^{\prime}\right) U\left(t^{\prime}, t_{0}\right)
$$

which on iteration leads to the perturbation series

$$
S=1+\sum_{N=1}^{\infty}(-i)^{N} \int_{-\infty}^{\infty} \mathrm{d} t_{1} \int_{-\infty}^{t_{1}} \mathrm{~d} t_{2} \cdots \int_{-\infty}^{t_{N-1}} \mathrm{~d} t_{N} H_{\text {int }}\left(t_{1}\right) \cdots H_{\text {int }}\left(t_{N}\right) .
$$

Since $H_{\text {int }}(t)$ given by Eq. (10) is linear in $\mathbf{A}$ (see Eq. (11)), the first-order $(N=1)$ term can contribute to either absorption through the first term in Eq. (11) or to emission through the second term in Eq. (11) and the integral over $\mathrm{d} t_{1}$, from $-\infty \rightarrow \infty$ leads to the respective energy conservation criteria of Bohr. In the scattering problem under consideration, the lowest order (in $e$ ) contribution to $\langle f|S| i\rangle$ is obtained from the $N=2$ term, which we may denote as $\left\langle f\left|S^{(2)}\right| i\right\rangle$. We introduce $\sum_{n}\left|\psi_{n}\right\rangle\left\langle\psi_{n}\right|=1$ between $H_{\text {int }}\left(t_{1}\right)$ and $H_{\text {int }}\left(t_{2}\right)$, neglect contribution from two photons in the intermediate state and employ the notation $|n\rangle=\left|\psi_{n}\right\rangle|\rangle_{0}$. This leads, on 
using Eqs. (11) and (12), to

$$
\begin{aligned}
& \left\langle n\left|H_{\text {int }}\left(t_{2}\right)\right| i\right\rangle=\mathscr{A}_{n i}\left(\mathbf{k}^{\prime}, \mu^{\prime}\right) \mathrm{e}^{\left[\mathrm{i}\left(E_{n}-E_{i}-\omega^{\prime}\right) t_{2}\right]}, \\
& \left\langle f\left|H_{\text {int }}\left(t_{1}\right)\right| n\right\rangle=\mathscr{E}_{f n}(\mathbf{k}, \mu) \mathrm{e}^{\left[\mathrm{i}\left(E_{f}+\omega-E_{n}\right) t_{1}\right]},
\end{aligned}
$$

where $\mathscr{A}_{n i}\left(\mathbf{k}^{\prime}, \mu^{\prime}\right)$ and $\mathscr{E} f n(\mathbf{k}, \mu)$ denote amplitudes for absorption and emission, involving $\mathbf{A}_{\mathbf{k}^{\prime} \mu^{\prime}}\left(\mathbf{r}_{j}\right)$ and $\mathbf{A}_{\mathbf{k} \mu}\left(\mathbf{r}_{j}\right)^{*}$ respectively, which are independent of time variable, instead of $\mathbf{A}\left(\mathbf{r}_{j}, t\right)$. We may change the variable of integration from $t_{2}$ to $t_{2}^{\prime}=t_{2}-t_{1}$, ranging from $-\infty \rightarrow 0$, associate a width $\Gamma_{n}$ with $\psi_{n}$ by introducing a factor $\exp \left(\Gamma_{n} t_{2}^{\prime}\right)($ see $[35,36])$ and obtain after completing both the integrations, the expression

$$
\left\langle f\left|S^{(2)}\right| i\right\rangle=2 \pi i \delta\left(E_{f}+\omega-E_{i}-\omega^{\prime}\right) T_{f i}\left(\mathbf{k} \mu ; \mathbf{k}^{\prime} \mu^{\prime}\right),
$$

where the on-energy-shell $T$-matrix element is of the form

$$
T_{f i}\left(\mathbf{k}, \mu ; \mathbf{k}^{\prime}, \mu^{\prime}\right)=\sum_{n} \mathscr{E}_{f n}(\mathbf{k}, \mu) \phi_{n} \mathscr{A}_{n i}\left(\mathbf{k}^{\prime}, \mu^{\prime}\right),
$$

and the profile function is given by

$$
\phi_{n}=\left(\omega_{n f}-\omega-i \Gamma_{n}\right)^{-1} ; \quad \omega_{n f}=E_{n}-E_{f},
$$

on making use of $E_{n}-E_{i}-\omega^{\prime}=\omega_{n i}-\omega^{\prime}=\omega_{n f}-\omega$ by virtue of the energy $\delta$-function in Eq. (20). Using Eq. (5) and observing that $\left\{\psi_{n}\right\}$ are completely antisymmetric with respect to the labels $1,2, \ldots, j, \ldots, Z$ of the electrons, we have

$$
\begin{aligned}
& \mathscr{A}_{n i}\left(\mathbf{k}^{\prime}, \mu^{\prime}\right)=\sum_{m_{n}^{\prime}, m_{i}^{\prime}} c_{m_{n}^{\prime}}^{s_{n}^{*}} c_{m_{i}^{\prime}}^{s_{i}}\left\langle J_{n} m_{n}^{\prime}\left|\mathscr{A}\left(\mathbf{k}^{\prime}, \mu^{\prime}\right)\right| J_{i} m_{i}^{\prime}\right\rangle ; \\
& \mathscr{E}_{f n}(\mathbf{k}, \mu)=\sum_{m_{f}^{\prime}, m_{n}^{\prime \prime}} c_{m_{f}^{\prime}}^{s_{f}^{*}} c_{m_{n}^{\prime \prime}}^{s_{n}}\left\langle J_{f} m_{f}^{\prime}|\mathscr{E}(\mathbf{k}, \mu)| J_{n} m_{n}^{\prime \prime}\right\rangle,
\end{aligned}
$$

where the matrix elements on the right-hand side satisfy

$$
\left\langle J_{\mathrm{u}} m_{\mathrm{u}}|\mathscr{A}(\mathbf{k}, \mu)| J_{1} m_{\mathrm{l}}\right\rangle=\left\langle J_{1} m_{\mathrm{l}}|\mathscr{E}(\mathbf{k}, \mu)| J_{\mathrm{u}} m_{\mathrm{u}}\right\rangle^{*}
$$

between any pair of lower and upper atomic states and

$$
\mathscr{E}(\mathbf{k}, \mu)=\frac{Z e}{(2 \pi)^{3 / 2} \sqrt{2 \omega}}\left[-i \mathbf{A}_{\mathbf{k}, \mu}^{*} \cdot \nabla+\frac{1}{2} \sigma \cdot\left(\nabla \times \mathbf{A}_{\mathbf{k}, \mu}^{*}\right)\right]
$$

with respect to an electron in the atom. Since atomic transitions during absorption and emission conserve total angular momentum and parity, we use the standard multipole expansion [26] for $\mathbf{A}_{\mathbf{k}, \mu}$ given by Eq. (13), viz,

$$
\mathbf{A}_{\mathbf{k}, \mu}=\mathrm{e}^{\mathrm{ik} \cdot \mathbf{r}} \hat{\varepsilon}_{\mu}=(2 \pi)^{1 / 2} \sum_{L=1}^{\infty} \sum_{M=-L}^{L}(i)^{L}[L] D_{M \mu}^{L}(\phi, \theta, 0)\left[\mathbf{A}_{L M}^{(m)}+i \mu \mathbf{A}_{L M}^{(e)}\right],
$$

where $[L]=(2 L+1)^{1 / 2}$ and $(\theta, \phi)$ denote polar angles of $\mathbf{k}$, while $\mathbf{A}_{L M}^{(m)}$ and $\mathbf{A}_{L M}^{(e)}$ denote, respectively, the 'magnetic' and 'electric' $2^{L}$-pole solutions of the free Maxwell equations. Using Eq. (26) we may write

$$
\mathscr{A}(\mathbf{k}, \mu)=\sum_{L=1}^{\infty} \sum_{M=-L}^{L} D_{M \mu}^{L}(\phi, \theta, 0)\left[\mathscr{J}_{L M}^{(m)}(\omega)+i \mu \mathscr{J}_{L M}^{(e)}(\omega)\right],
$$

where the notation

$$
\mathscr{J}_{L M}^{(m / e)}(\omega)=\frac{Z e i^{L}[L]}{2 \pi \sqrt{2 \omega}}\left[-i \mathbf{A}_{L M}^{(m / e)} \cdot \nabla+\frac{1}{2} \sigma \cdot\left(\nabla \times \mathbf{A}_{L M}^{(m / e)}\right)\right]
$$

is used. Noting that $\mathscr{J}_{L M}^{(m / e)}(\omega)$ is an irreducible tensor of rank $L$, we may apply the Wigner-Eckart theorem to write

$$
\left\langle J_{\mathrm{u}} m_{\mathrm{u}}\left|\mathscr{J}_{L M}^{(m / e)}(\omega)\right| J_{1} m_{1}\right\rangle=C\left(J_{1}, L, J_{\mathrm{u}} ; m_{1}, M, m_{\mathrm{u}}\right)\left\langle J_{\mathrm{u}}\left\|\mathscr{J}_{L M}^{(m / e)}(\omega)\right\| J_{1}\right\rangle .
$$


Thus we obtain

$$
\left\langle J_{\mathrm{u}} m_{\mathrm{u}}|\mathscr{A}(\mathbf{k}, \mu)| J_{1} m_{\mathrm{l}}\right\rangle=\mathscr{A}(k, \mu)_{m_{\mathrm{u}} m_{\mathrm{l}}}=\sum_{L} C\left(J_{1}, L, J_{\mathrm{u}} ; m_{\mathrm{l}}, M, m_{\mathrm{u}}\right) \mathscr{J}_{L}(\omega)(i \mu)^{g_{+}(L)} D_{M \mu}^{L}(\phi, \theta, 0),
$$

where the reduced matrix elements are given by

$$
\mathscr{J}_{L}(\omega)=\left\langle J_{\mathrm{u}}\left\|\mathscr{J}_{L}^{(m)}(\omega)\right\| J_{1}\right\rangle g_{-}(L)+\left\langle J_{\mathrm{u}}\left\|\mathscr{J}_{L}^{(e)}(\omega)\right\| J_{1}\right\rangle g_{+}(L),
$$

in terms of the projection operators

$$
g_{ \pm}(L)=\frac{1}{2}\left[1 \pm(-1)^{L} \pi_{\mathrm{u}} \pi_{1}\right] .
$$

In the above equation $\pi_{\mathrm{u}}, \pi_{1}$ denote the parities of the upper and lower levels. Using Eq. (24), we have

$$
\left\langle J_{1} m_{1}|\mathscr{E}(\mathbf{k}, \mu)| J_{\mathrm{u}} m_{\mathrm{u}}\right\rangle=\mathscr{E}(\mathbf{k}, \mu)_{m_{\mathrm{l}} m_{\mathrm{u}}}=\sum_{L} C\left(J_{1}, L, J_{\mathrm{u}} ; m_{1}, M, m_{\mathrm{u}}\right) \mathscr{J}_{L}(\omega)^{*}(-i \mu)^{g_{+}(L)} D_{M \mu}^{L}(\phi, \theta, 0)^{*} .
$$

Thus, we may express Eq. (21) as

$$
T_{f i}\left(\mathbf{k} \mu ; \mathbf{k}^{\prime} \mu^{\prime}\right)=\sum_{n} \phi_{n} \sum_{m_{f}^{\prime} m_{i}^{\prime}} c_{m_{f}^{\prime}}^{s_{f}^{*}} c_{m_{i}^{\prime}}^{s_{i}}\left[\mathscr{E}(\mathbf{k}, \mu) \mathscr{G}^{S_{n}} \mathscr{A}\left(\mathbf{k}^{\prime}, \mu^{\prime}\right)\right]_{m_{f}^{\prime} m_{i}^{\prime}},
$$

where the summation $\sum_{n}$ implies summation with respect to $s_{n}$ as well, and $\mathscr{A}\left(\mathbf{k}^{\prime}, \mu^{\prime}\right)$ and $\mathscr{E}(\mathbf{k}, \mu)$ denote matrices, whose elements

$$
\begin{aligned}
& \left\langle J_{n} m_{n}^{\prime}\left|\mathscr{A}\left(\mathbf{k}^{\prime}, \mu^{\prime}\right)\right| J_{i} m_{i}^{\prime}\right\rangle=\mathscr{A}\left(\mathbf{k}^{\prime}, \mu^{\prime}\right)_{m_{n}^{\prime} m_{i}^{\prime}} ; \\
& \left\langle J_{f} m_{f}^{\prime}|\mathscr{E}(\mathbf{k}, \mu)| J_{n} m_{n}^{\prime \prime}\right\rangle=\mathscr{E}(\mathbf{k}, \mu)_{m_{f}^{\prime} m_{n}^{\prime \prime}},
\end{aligned}
$$

may be written explicitly using Eqs. (30) and (33) and $\mathscr{G}^{S_{n}}$ denotes a hermitian $\left(2 J_{n}+1\right) \times\left(2 J_{n}+1\right)$ matrix, which is defined in terms of its elements

$$
\mathscr{G}_{m_{n}^{\prime \prime} m_{n}^{\prime}}^{s_{n}}=c_{m_{n}^{\prime \prime}}^{s_{n}} c_{m_{n}^{\prime}}^{s_{n}^{*}} \text {. }
$$

Clearly, the summation over $n$ on right-hand side of Eq. (34) indicates a summation with respect to all the atomic states $\left\{\psi_{n}\right\}$, which constitute the complete orthogonal set. Since $n$ is a cumulative index $\sum_{n}$ includes $\sum_{J_{n}} \sum_{S_{n}=1}^{2 J_{n}+1}$, apart from summation with respect to other quantum numbers. The left-hand side of Eq. (34) is written for a given $\psi_{i}$ and $\psi_{f}$ with energies $E_{i}$ and $E_{f}$, respectively. The quantities $s_{i}$ and $s_{f}$ are specified by lefthand side of Eq. (34) and hence they are fixed entities on right-hand side of Eq. (34).

In the absence of the electric quadrupole field, the $s_{f}, s_{n}, s_{i}$ may be replaced, respectively, by appropriate $M_{f}, M_{n}, M_{i}$ which are determined by the Kronecker $\delta$-function in Eq. (7), when the magnetic field $\mathbf{B}$ alone is present and is directed along $\left(\theta_{B}, \phi_{B}\right)$. Thus, $c_{m_{f}^{\prime}}^{s_{f}^{*}}$ and $c_{m_{i}^{\prime}}^{s_{i}}$ are replaced, respectively, by $D_{m_{f}^{\prime} M_{f}}^{J_{f}}\left(\phi_{B}, \theta_{B}, 0\right)^{*}$ and $D_{m_{i}^{\prime} M_{i}}^{J_{i}}\left(\phi_{B}, \theta_{B}, 0\right)$ with $M_{f}$ and $M_{i}$ being fixed by left-hand side. It may be noted that $\phi_{n}$ depends on $M_{n}$ and the summation over $n$ includes $\sum_{J_{n}} \sum_{M_{n}=-J_{n}}^{J_{n}}$, with $\mathscr{G}^{S_{n}}$ replaced now by $\mathscr{G}^{M_{n}}$ whose elements are given by

$$
\mathscr{G}_{m_{n}^{\prime \prime} m_{n}^{\prime}}^{M_{n}}=D_{m_{n}^{\prime \prime} M_{n}}^{J_{n}}(\phi, \theta, 0) D_{m_{n}^{\prime} M_{n}}^{J_{n}}(\phi, \theta, 0)^{*} .
$$

If the magnetic field $\mathbf{B}$ is along the $Z$-axis of ARF itself, the $s_{f}, s_{n}, s_{i}$ in Eq. (34) may, respectively, be replaced by $m_{f}, m_{n}, m_{i}$ determined by the Kronecker $\delta$-function in Eq. (8). Thus, $c_{m_{f}^{\prime}}^{s_{*}^{*}}$ and $c_{m_{i}^{\prime}}^{s_{i}}$ are replaced, respectively, by $\delta_{m_{f}^{\prime} m_{f}}$ and $\delta_{m_{i}^{\prime} m_{i}}$, where $m_{f}$ and $m_{i}$ are fixed by left-hand side of Eq. (34). Therefore, the summation with respect to $m_{f}^{\prime}$ and $m_{i}^{\prime}$ on right-hand side of Eq. (34) drops after making the replacements $c_{m_{f}^{\prime}}^{s_{f}}=1$ and $c_{m_{i}^{\prime}}^{s_{i}}=1$. The $\psi_{n}$ depends on $m_{n}$ and the summation over $n$ includes $\sum_{J_{n}} \sum_{m_{n}=-J_{n}}^{J_{n}}$, with $\mathscr{G}^{s_{n}}$ replaced by $\mathscr{G}^{m_{n}}$, whose elements are given by

$$
\mathscr{G}_{m_{n}^{\prime \prime} m_{n}^{\prime}}^{m_{n}}=\delta_{m_{n}^{\prime \prime} m_{n}} \delta_{m_{n}^{\prime} m_{n}},
$$

i.e., $\mathscr{G}^{S_{n}}$ gets replaced by a diagonal matrix with zeros everywhere except $\mathscr{G}_{m_{n} m_{n}}^{m_{n}}=1$ in Eq. (34). 
It may be noted that the atomic transitions from $\psi_{i}$ to $\psi_{n}$ following absorption of $\omega^{\prime}$ and from $\psi_{n}$ to $\psi_{f}$ consequent to the emission of $\omega$ are virtual transitions, which do not satisfy the celebrated Bohr criteria. This is in contrast to absorption or emission represented by the $N=1$ term. They are real transitions which satisfy the Bohr criteria as already pointed out. The summation over $n$ includes all atomic states $\psi_{n}$ with different energy eigenvalues $E_{n}$. However, all of them do not contribute equally to Eq. (34). The presence of $\phi_{n}$ on right-hand side of Eq. (34) indicates that one has to pay more attention to contributions coming from those states $\psi_{n}$ with $E_{n}$ close to $E_{i}+\omega^{\prime}=E_{f}+\omega$. If there is an $E_{n}$ such that $E_{n}=E_{i}+\omega^{\prime}=E_{f}+\omega$, the contribution from this state alone overshadows all other contributions. The scattering is then referred to as resonance scattering. In particular, if $E_{i}=E_{f}$ the terminology 'two-level resonance scattering' is employed. This is shown as (a) in Fig. 2, where $\omega^{\prime}=\omega$. On the other hand, if $E_{f}>E_{i}$ as in (b) of Fig. 2, the resonance scattering is referred to as three-level resonance or fluorescence.

If there is no electric quadrupole field and the atom is exposed only to a pure magnetic field $\mathbf{B}$, such that the $\left(2 J_{n}+1\right)$ states $\left|J_{n} M_{n}\right\rangle$ refer to distinctly separated energy levels as in Zeeman effect, one can envisage resonance scattering taking place individually with each one of these taking the role of the upper level, as shown in (a) and (b) of Fig. 2, if the condition $E_{M_{n}}=E_{i}+\omega^{\prime}=E_{f}+\omega$ is satisfied. On the other hand, if $g B<\Gamma_{n}$, the levels are not distinct and all of them contribute coherently to form a single line. This is referred to as quantum interference in the context of Hanle scattering, which is shown as (c) in Fig. 2. In contrast to Hanle effect where interference occurs between magnetic substates with the same $J_{n}$, interference effects between states with different $J_{n}$ have also been observed in polarization studies of solar Ca II H-K and Na I $\mathrm{D}_{1}$ and $\mathrm{D}_{2}$ lines [13], wherein it is mentioned that this can take place even when the lines are $3.5 \mathrm{~nm}$ apart. The general terminology, 'Raman scattering' has been employed [12,13] to denote scattering, where contributions from several intermediate states are involved. In general, therefore, we may rewrite Eq. (21) in the form

$$
T_{f i}\left(\mathbf{k}, \mu ; \mathbf{k}^{\prime}, \mu^{\prime}\right)=\left\langle\psi_{f}|\mathscr{E}(\mathbf{k}, \mu)| \psi_{v}\right\rangle,
$$

where $\left|\psi_{v}\right\rangle$ represents a virtual state defined by

$$
\left|\psi_{v}\right\rangle=\sum_{n} c_{n}^{v}\left|\psi_{n}\right\rangle ; \quad\left|c_{n}^{v}\right\rangle=\phi_{n}\left\langle\psi_{n}\left|\mathscr{A}\left(\mathbf{k}^{\prime}, \mu^{\prime}\right)\right| \psi_{i}\right\rangle
$$

a

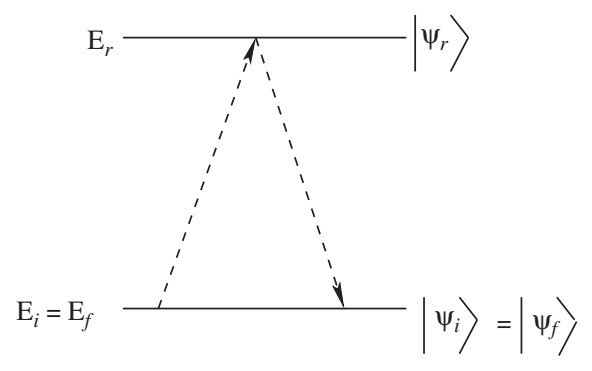

C

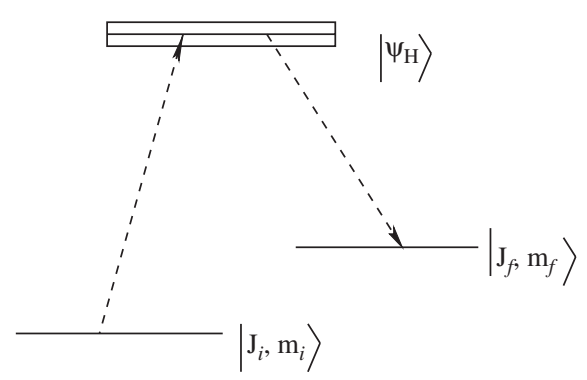

b

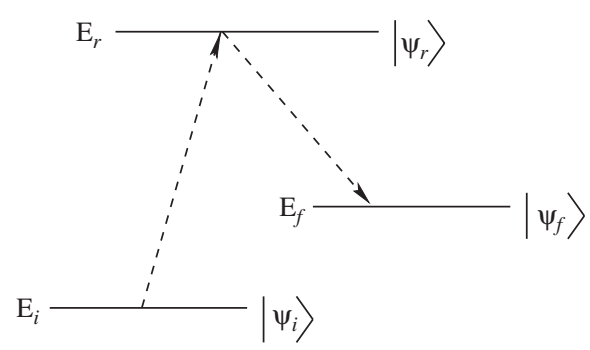

d

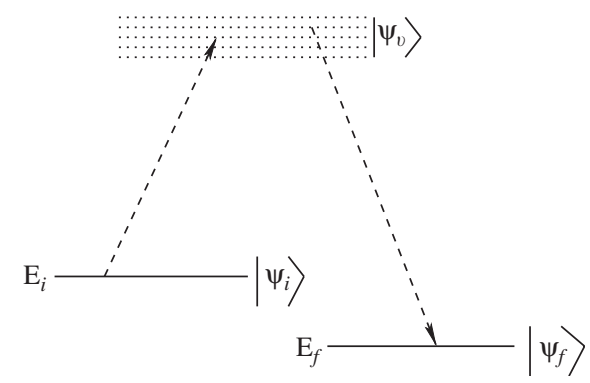

Fig. 2. Level diagrams showing the atom-radiation interaction processes discussed in this paper. (a) Two-level resonance scattering process, (b) three-level fluorescence scattering process, (c) Hanle scattering process in weak magnetic fields and (d) the general case of Raman scattering. 
which is clearly not an eigenstate of energy. In Raman effect, shown as (d) in Fig. 2, the lines corresponding to $E_{f}<E_{i}$ are referred to as anti-Stokes lines, in contrast to those with $E_{f}>E_{i}$ referred to as Stokes lines.

\subsection{The dipole approximation}

If we neglect the spin-dependent second term in Eq. (25) and employ dipole approximation $\mathrm{e}^{\mathrm{ik} \cdot \mathbf{r}} \approx 1$ in $\mathbf{A}_{\mathbf{k} \mu}(\mathbf{r})$ given by Eq. (13), then we may express Eq. (24) as

$$
\left\langle\psi_{\mathrm{u}}|\mathscr{A}(\mathbf{k}, \mu)| \psi_{1}\right\rangle \approx\left\langle\psi_{\mathrm{u}}\left|\hat{\varepsilon}_{\mu} \cdot \mathbf{p}\right| \psi_{1}\right\rangle \approx\left\langle\psi_{1}|\mathscr{E}(\mathbf{k}, \mu)| \psi_{\mathrm{u}}\right\rangle^{*} .
$$

In the above equation the momentum operator $\mathbf{p}=-i \nabla$ may be replaced by $\left[\mathbf{r}, H_{0}\right]$, to obtain

$$
\left\langle\psi_{\mathrm{u}}\left|\hat{\varepsilon}_{\mu} \cdot\left[\mathbf{r}, H_{0}\right]\right| \psi_{1}\right\rangle \approx\left(E_{1}-E_{\mathrm{u}}\right)\left\langle\psi_{\mathrm{u}}\left|\left(\hat{\varepsilon}_{\mu} \cdot \mathbf{r}\right)\right| \psi_{1}\right\rangle,
$$

if $E_{\mathrm{u}}, E_{1}$ denote the energy eigenvalues of $\psi_{\mathrm{u}}, \psi_{1}$ when considered as eigenstates of Eq. (1). We, thus, realize the Kramers-Heisenberg form represented by Eq. (1) of [14].

\section{The scattering matrix for atoms in external electric quadrupole and uniform magnetic fields}

The central result of the previous section is the derivation of the general expression for the on-energy-shell $T$-matrix element $T_{f i}\left(\mu, \mu^{\prime}\right)$. If the incident radiation is in a pure state

$$
\hat{\varepsilon}_{i}=\sum_{\mu^{\prime}} c_{\mu^{\prime}}^{i} \hat{\varepsilon}_{\mu^{\prime}}^{\prime}
$$

the amplitude for detecting the scattered radiation in a pure state

$$
\hat{\varepsilon}_{f}=\sum_{\mu} c_{\mu}^{f} \hat{\varepsilon}_{\mu}
$$

is given by

$$
T_{f i}\left(\hat{\varepsilon}_{f}, \hat{\varepsilon}_{i}\right)=\sum_{\mu \mu^{\prime}} c_{\mu}^{f^{*}} c_{\mu^{\prime}}^{i} T_{f i}\left(\mu, \mu^{\prime}\right)
$$

where $\sum_{\mu^{\prime}}\left|c_{\mu^{\prime}}^{i}\right|^{2}=1=\sum_{\mu}\left|c_{\mu}^{f}\right|^{2}$. On the other hand, it is more convenient to employ the density matrix formalism $[24,25,37]$ to describe the states of polarization of the incident and scattered radiation, as it is more general and can handle mixed states of polarization as well.

\subsection{The density matrix for polarized radiation}

The density matrix $\rho$ for polarized radiation may be written as

$$
\rho=\frac{1}{2}\left[I+\sigma_{x}^{\gamma} Q+\sigma_{y}^{\gamma} U+\sigma_{z}^{\gamma} V\right]=\frac{1}{2} \sum_{p=0}^{3} \sigma_{p}^{\gamma} S_{p},
$$

in terms of the well-known [2] Stokes parameters $\left(I=S_{0}, Q=S_{1}, U=S_{2}, V=S_{3}\right)$ and Pauli matrices $\sigma_{x}^{\gamma}=$ $\sigma_{1}^{\gamma}, \sigma_{y}^{\gamma}=\sigma_{2}^{\gamma}, \sigma_{z}^{\gamma}=\sigma_{3}^{\gamma}$ and the unit matrix $\sigma_{0}^{\gamma}$ whose rows and columns are labeled by the left and right circular polarization states $|\mu= \pm 1\rangle$ of radiation. Clearly,

$$
S_{p}=\operatorname{tr}\left(\sigma_{p}^{\gamma} \rho\right), \quad p=0,1,2,3,
$$

where $\operatorname{tr}$ denotes the trace or spur. A column vector $\mathbf{S}$ with elements $S_{p}, p=0,1,2,3$ is referred to as the Stokes vector for polarization. If we consider $T$ in Eq. (21) as a $2 \times 2$ matrix $T$ with elements $T_{\mu \mu^{\prime}} \equiv T_{f i}\left(\mu, \mu^{\prime}\right)$, the density matrix $\rho$ of scattered radiation is given by

$$
\rho=T \rho^{\prime} T^{\dagger},
$$


where $\rho^{\prime}$ denotes the density matrix of polarized radiation incident on the atom. Using Eq. (47), we have

$$
S_{p}=\frac{1}{2} \sum_{p^{\prime}=0}^{3} \operatorname{tr}\left(\sigma_{p}^{\gamma} T \sigma_{p^{\prime}}^{\gamma} T^{\dagger}\right) S_{p^{\prime}}^{\prime}
$$

for the Stokes parameters of the scattered radiation, in terms of the matrix $T$, its hermitian conjugate $T^{\dagger}$ and the Stokes parameters $S_{p^{\prime}}^{\prime}$ characterizing the radiation incident on the atom.

\subsection{The scattering matrix}

If the Stokes vector $\mathbf{S}^{\prime}$ with elements $\left(I^{\prime}=S_{0}^{\prime}, Q^{\prime}=S_{1}^{\prime}, U^{\prime}=S_{2}^{\prime}, V^{\prime}=S_{3}^{\prime}\right)$, characterizes the radiation incident on the atom, the Stokes vector $\mathbf{S}$ characterizing the scattered radiation may be expressed as

$$
\mathbf{S}=\mathscr{R} \mathbf{S}^{\prime},
$$

where the $4 \times 4$ matrix $\mathscr{R}$ is referred to as the scattering matrix. Comparison of Eqs. (49) and (50) readily identifies the elements of $\mathscr{R}$ as

$$
\mathscr{R}_{p p^{\prime}}=\frac{1}{2} \sum_{\substack{\mu \mu^{\prime} \\ \mu^{\prime \prime} \mu^{\prime \prime \prime}}}\left(\sigma_{p}^{\gamma}\right)_{\mu^{\prime \prime} \mu} T_{\mu \mu^{\prime}}\left(\sigma_{p^{\prime}}^{\gamma}\right)_{\mu^{\prime} \mu^{\prime \prime \prime}}\left(T^{\dagger}\right)_{\mu^{\prime \prime \prime} \mu^{\prime \prime}}
$$

where we may use Eq. (34) for $T_{\mu \mu^{\prime}}$ and note that $\left(T^{\dagger}\right)_{\mu^{\prime \prime \prime} \mu^{\prime \prime}}=T_{\mu^{\prime \prime} \mu^{\prime \prime \prime}}^{*}$, for which we may use the complex conjugate of Eq. (34). We may thus write

$$
\begin{aligned}
& T_{\mu \mu^{\prime}}=\sum_{n} \phi_{n} \sum_{m_{f}^{\prime} m_{i}^{\prime}} c_{m_{f}^{\prime}}^{s_{f}^{*}} c_{m_{i}^{\prime}}^{s_{i}} \mathscr{M}_{m_{f}^{\prime} m_{i}^{\prime}}\left(\mu, \mu^{\prime}\right) ; \\
& \left(T^{\dagger}\right)_{\mu^{\prime \prime \prime} \mu^{\prime \prime}}=T_{\mu^{\prime \prime} \mu^{\prime \prime \prime}}^{*}=\sum_{n^{\prime}} \phi_{n^{\prime}}^{*} \sum_{m_{f}^{\prime \prime} m_{i}^{\prime \prime}} c_{m_{f}^{\prime \prime}}^{s_{f}} c_{m_{i}^{\prime \prime}}^{s_{i}^{*}} \mathscr{M}_{m_{f}^{\prime \prime} m_{i}^{\prime \prime}}\left(\mu^{\prime \prime}, \mu^{\prime \prime \prime}\right)^{*},
\end{aligned}
$$

where

$$
\begin{aligned}
& \mathscr{M}_{m_{f}^{\prime} m_{i}^{\prime}}\left(\mu, \mu^{\prime}\right)=\left[\mathscr{E}(\mathbf{k}, \mu) \mathscr{G}^{S_{n}} \mathscr{A}\left(\mathbf{k}^{\prime}, \mu^{\prime}\right)\right]_{m_{f}^{\prime} m_{i}^{\prime}} ; \\
& \mathscr{M}_{m_{f}^{\prime \prime} m_{i}^{\prime \prime}}\left(\mu^{\prime \prime}, \mu^{\prime \prime \prime}\right)^{*}=\left[\mathscr{E}\left(\mathbf{k}, \mu^{\prime \prime}\right) \mathscr{G}^{S_{n^{\prime}}} \mathscr{A}\left(\mathbf{k}^{\prime}, \mu^{\prime \prime \prime}\right)\right]_{m_{f}^{\prime \prime} m_{i}^{\prime \prime}}^{*}=\left[\mathscr{A}^{\dagger}\left(\mathbf{k}^{\prime}, \mu^{\prime \prime \prime}\right) \mathscr{G}^{S_{n^{\prime}}} \mathscr{E}^{\dagger}\left(\mathbf{k}, \mu^{\prime \prime}\right)\right]_{m_{i}^{\prime \prime} m_{f}^{\prime \prime}},
\end{aligned}
$$

since $\mathscr{G}_{n^{\prime}}$ is hermitian. Using the above in Eq. (51), we have

$$
\begin{aligned}
\mathscr{R}_{p p^{\prime}}= & \frac{1}{2} \sum_{\substack{\mu \mu^{\prime} \\
\mu^{\prime \prime} \mu^{\prime \prime \prime}}}\left(\sigma_{p}^{\gamma}\right)_{\mu^{\prime \prime} \mu}\left(\sigma_{p^{\prime}}^{\gamma}\right)_{\mu^{\prime} \mu^{\prime \prime \prime}} \sum_{n n^{\prime}} \phi_{n} \phi_{n^{\prime}}^{*} \sum_{\substack{m_{f}^{\prime} m_{i}^{\prime} \\
m_{f}^{\prime \prime} m_{i}^{\prime \prime}}}\left[\mathscr{E}(\mathbf{k}, \mu) \mathscr{G}^{S_{n}} \mathscr{A}\left(\mathbf{k}^{\prime}, \mu^{\prime}\right)\right]_{m_{f}^{\prime} m_{i}^{\prime}} \\
& \times c_{m_{i}^{\prime}}^{s_{i}} c_{m_{i}^{\prime \prime}}^{s_{i}^{*}}\left[\mathscr{A}^{\dagger}\left(\mathbf{k}^{\prime}, \mu^{\prime \prime \prime}\right) \mathscr{G}^{S_{n^{\prime}}} \mathscr{E}^{\dagger}\left(\mathbf{k}, \mu^{\prime \prime}\right)\right]_{m_{i}^{\prime \prime} m_{f}^{\prime \prime}} c_{m_{f}^{\prime \prime}}^{s_{f}} c_{m_{f}^{\prime}}^{s_{f}^{*}}
\end{aligned}
$$

Following Eq. (36), we may define hermitian matrices $\mathscr{G}^{s_{i}}$ and $\mathscr{G}^{s_{f}}$ through their elements

$$
\begin{gathered}
\mathscr{G}_{m_{i}^{\prime} m_{i}^{\prime \prime}}^{s_{i}}=c_{m_{i}^{\prime}}^{s_{i}} c_{m_{i}^{\prime \prime}}^{s_{i}^{*}}, \\
\mathscr{G}_{m_{f}^{\prime \prime} m_{f}^{\prime}}^{s_{f}}=c_{m_{f}^{\prime \prime}}^{s_{f}} c_{m_{f}^{\prime}}^{s_{f}^{*}},
\end{gathered}
$$

so that we may rewrite Eq. (54) as

$$
\mathscr{R}_{p p^{\prime}}=\frac{1}{2} \sum_{\substack{\mu \mu^{\prime} \\ \mu^{\prime \prime} \mu^{\prime \prime \prime}}}\left(\sigma_{p}^{\gamma}\right)_{\mu^{\prime \prime} \mu}\left(\sigma_{p^{\prime}}^{\gamma}\right)_{\mu^{\prime} \mu^{\prime \prime \prime}} \sum_{n n^{\prime}} \phi_{n} \phi_{n^{\prime}}^{*} \operatorname{Tr}\left[\mathscr{E}(\mathbf{k}, \mu) \mathscr{G}^{S_{n}} \mathscr{A}\left(\mathbf{k}^{\prime}, \mu^{\prime}\right) \mathscr{G}^{S_{i}} \mathscr{A}^{\dagger}\left(\mathbf{k}^{\prime}, \mu^{\prime \prime \prime}\right) \mathscr{G}^{s_{n^{\prime}}} \mathscr{E}^{\dagger}\left(\mathbf{k}, \mu^{\prime \prime}\right) \mathscr{G}^{S_{f}}\right],
$$

where $\operatorname{Tr} \equiv \sum_{m_{f}^{\prime}}$ denotes the trace or spur of the $\left(2 J_{f}+1\right) \times\left(2 J_{f}+1\right)$ matrix within the square brackets, which is defined through matrix multiplication of the eight matrices, each of which is well defined through 
Eqs. (30), (33), (36), (55), (56)) for any specified atomic transition from an initial state $\psi_{i}$ with energy $E_{i}$ and total angular momentum $J_{i}$ to a final state $\psi_{f}$ with energy $E_{f}$ and total angular momentum $J_{f}$, when the atom is exposed to a combined external electric quadrupole field and a uniform magnetic field. It may be noted that $s_{i}$ and $s_{f}$ are fixed and the summation over $n, n^{\prime}$ includes summation over $s_{n}, s_{n^{\prime}}$.

\subsection{The particular case of resonance scattering via electric dipole transitions between $J_{i}=J_{f}=0$ and $J_{n}=1$}

In this important particular case, which has often been investigated in the presence of pure magnetic fields, it is clear that $\mathscr{G}^{S_{i}}=\mathscr{G}^{S_{f}}=1$ in Eq. (57) and $L=L^{\prime}=1$ in Eqs. (30) and (33), so that we may write the trace appearing in Eq. (57) as

$$
\begin{aligned}
\operatorname{tr} & {\left[\mathscr{A}\left(\mathbf{k}^{\prime}, \mu^{\prime}\right) \mathscr{A}^{\dagger}\left(\mathbf{k}^{\prime}, \mu^{\prime \prime \prime}\right) \mathscr{G}^{S_{n^{\prime}}} \mathscr{E}^{\dagger}\left(\mathbf{k}, \mu^{\prime \prime}\right) \mathscr{E}(\mathbf{k}, \mu) \mathscr{G}^{S_{n}}\right] } \\
& =\sum_{\substack{m^{\prime} m^{\prime \prime \prime} \\
m_{n}^{\prime \prime \prime} m_{n}^{\prime \prime \prime}}}\left[\mathscr{A}\left(\mathbf{k}^{\prime}, \mu^{\prime}\right) \mathscr{A}^{\dagger}\left(\mathbf{k}^{\prime}, \mu^{\prime \prime \prime}\right)\right]_{m_{n}^{\prime} m_{n}^{\prime \prime \prime}}\left(\mathscr{G}^{S_{n^{\prime}}}\right)_{m_{n}^{\prime \prime \prime} m_{n}^{\prime \prime \prime}}\left[\mathscr{E}^{\dagger}\left(\mathbf{k}, \mu^{\prime \prime}\right) \mathscr{E}(\mathbf{k}, \mu)\right]_{m_{n}^{\prime \prime \prime} m_{n}^{\prime \prime}}\left(\mathscr{G}^{S_{n}}\right)_{m_{n}^{\prime \prime} m_{n}^{\prime}} .
\end{aligned}
$$

We may use Eq. (30), in combination with Eq. (35), to write

$$
\left[\mathscr{A}\left(\mathbf{k}^{\prime}, \mu^{\prime}\right) \mathscr{A}^{\dagger}\left(\mathbf{k}^{\prime}, \mu^{\prime \prime \prime}\right)\right]_{m_{n}^{\prime} m_{n}^{\prime \prime \prime}}=\left|\mathscr{J}_{1}\left(\omega^{\prime}\right)\right|^{2} \mu^{\prime} \mu^{\prime \prime \prime} D_{m_{n}^{\prime} \mu^{\prime}}^{1}\left(\phi^{\prime}, \theta^{\prime}, 0\right) D_{m_{n}^{\prime \prime \prime} \mu^{\prime \prime \prime}}^{1}\left(\phi^{\prime}, \theta^{\prime}, 0\right)^{*},
$$

and Eq. (33), in combination with Eq. (35), to write

$$
\left[\mathscr{E}^{\dagger}\left(\mathbf{k}, \mu^{\prime \prime}\right) \mathscr{E}(\mathbf{k}, \mu)\right]_{m_{n}^{\prime \prime \prime} m_{n}^{\prime \prime}}=\left|\mathscr{J}_{1}(\omega)\right|^{2} \mu \mu^{\prime \prime} D_{m_{n}^{\prime \prime \prime} \mu^{\prime \prime}}^{1}(\phi, \theta, 0) D_{m_{n}^{\prime \prime} \mu}^{1}(\phi, \theta, 0)^{*}
$$

Using Eq. (36) for $\left(\mathscr{G}^{S_{n}}\right)_{m_{n}^{\prime \prime} m_{n}^{\prime}}$ and $\left(\mathscr{G}^{S_{n^{\prime}}}\right)_{m_{n}^{\prime \prime \prime} m_{n}^{\prime \prime \prime}}$, we may attach $c_{m_{n}^{\prime}}^{s_{n}^{*}} c_{m_{n}^{\prime \prime \prime}}^{s_{n^{\prime}}}$ along with $\left(\sigma_{p^{\prime}}^{\gamma}\right)_{\mu^{\prime} \mu^{\prime \prime \prime}}$ to Eq. (59), while we may attach $c_{m_{n}^{\prime \prime}}^{s_{n}} c_{m_{n}^{\prime \prime \prime}}^{s_{n^{\prime \prime}}^{*}}$ along with $\left(\sigma_{p}^{\gamma}\right)_{\mu^{\prime \prime} \mu}$ to Eq. (60), so that

$$
\begin{aligned}
& \sum_{\mu^{\prime} \mu^{\prime \prime \prime}}\left(\sigma_{p^{\prime}}^{\gamma}\right)_{\mu^{\prime} \mu^{\prime \prime \prime}} \mu^{\prime} \mu^{\prime \prime \prime} \sum_{m_{n}^{\prime} m_{n}^{\prime \prime \prime}} c_{m_{n}^{\prime}}^{s_{n}^{*}} c_{m_{n}^{\prime \prime \prime}}^{s_{n^{\prime}}} D_{m_{n}^{\prime} \mu^{\prime}}^{1}\left(\phi^{\prime}, \theta^{\prime}, 0\right) D_{m_{n}^{\prime \prime \prime} \mu^{\prime \prime \prime}}^{1}\left(\phi^{\prime}, \theta^{\prime}, 0\right)^{*} \\
& =\sum_{\mu_{a}=-\lambda_{a}}^{\lambda_{a}} \sum_{m_{a}=-\lambda_{a}}^{\lambda_{a}} f_{p^{\prime}}\left(\lambda_{a}, \mu_{a}\right) F_{n n^{\prime}}\left(\lambda_{a}, m_{a}\right) D_{m_{a} \mu_{a}}^{\lambda_{a}}\left(\phi^{\prime}, \theta^{\prime}, 0\right) \\
& \sum_{\mu \mu^{\prime \prime}}\left(\sigma_{p}^{\gamma}\right)_{\mu^{\prime \prime} \mu} \mu^{\prime \prime} \mu \sum_{m_{n}^{\prime \prime} m_{n}^{\prime \prime \prime}} c_{m_{n}^{\prime \prime}}^{s_{n}} c_{m_{n}^{\prime \prime \prime}}^{s_{n^{\prime}}^{*}} D_{m_{n}^{\prime \prime \prime} \mu^{\prime \prime}}^{1}(\phi, \theta, 0) D_{m_{n}^{\prime \prime} \mu}^{1}(\phi, \theta, 0)^{*} \\
& =\sum_{\lambda_{e}=0}^{2} \sum_{\mu_{e}=-\lambda_{e}}^{\lambda_{e}} \sum_{m_{e}=-\lambda_{e}}^{\lambda_{e}} f_{p}\left(\lambda_{e}, \mu_{e}\right) F_{n, n^{\prime}}\left(\lambda_{e}, m_{e}\right)^{*} D_{m_{e} \mu_{e}}^{\lambda_{e}}(\phi, \theta, 0) .
\end{aligned}
$$

Thus, we have

$$
\begin{aligned}
\mathscr{R}_{p p^{\prime}}= & \frac{1}{2}\left|\mathscr{J}_{1}(\omega)\right|^{2}\left|\mathscr{J}_{1}\left(\omega^{\prime}\right)\right|^{2} \sum_{n n^{\prime}} \phi_{n} \phi_{n^{\prime}}^{*} \sum_{\lambda_{a}=0}^{2} \sum_{\lambda_{e}=0}^{2} F_{n, n^{\prime}}\left(\lambda_{a}, m_{a}\right) F_{n, n^{\prime}}\left(\lambda_{e}, m_{e}\right)^{*} \\
& \times f_{p}\left(\lambda_{e}, \mu_{e}\right) f_{p^{\prime}}\left(\lambda_{a}, \mu_{a}\right) D_{m_{e} \mu_{e}}^{\lambda_{e}}(\phi, \theta, 0) D_{m_{a} \mu_{a}}^{\lambda_{a}}\left(\phi^{\prime}, \theta^{\prime}, 0\right),
\end{aligned}
$$

where

$$
\begin{aligned}
& F_{n, n^{\prime}}(\lambda, m)=\sum_{m_{n}^{\prime}} C\left(1,1, \lambda ; m_{n}^{\prime},-m_{n}^{\prime \prime \prime}, m\right)(-1)^{m_{n}^{\prime \prime \prime}} c_{m_{n}^{\prime}}^{s_{n}^{*}} c_{m_{n}^{\prime \prime \prime}}^{s_{n^{\prime}}} \\
& f_{p}(\lambda, \mu)=\sum_{\lambda} C\left(1,1, \lambda ; \mu^{\prime},-\mu^{\prime \prime \prime}, \mu\right)(-1)^{-\mu^{\prime \prime \prime}} \mu^{\prime} \mu^{\prime \prime \prime}\left(\sigma_{p}^{\gamma}\right)_{\mu^{\prime} \mu^{\prime \prime \prime}}
\end{aligned}
$$


The $f_{p}(\lambda, \mu)$ for $p=0,1,2,3$ may explicitly be written as

$$
\begin{aligned}
& f_{0}(\lambda, \mu)=\frac{2}{\sqrt{3}}\left[\delta_{\lambda, 0}+\frac{1}{\sqrt{2}} \delta_{\lambda, 2}\right] \delta_{\mu, 0} ; \\
& f_{1}(\lambda, \mu)=-\delta_{\lambda, 2}\left[\delta_{\mu, 2}+\delta_{\mu,-2}\right] ; \\
& f_{2}(\lambda, \mu)=i \delta_{\lambda, 2}\left[\delta_{\mu, 2}-\delta_{\mu,-2}\right] ; \\
& f_{3}(\lambda, \mu)=\sqrt{2} \delta_{\lambda, 1} \delta_{\mu, 0} .
\end{aligned}
$$

It is interesting to note that $F_{n, n^{\prime}}(\lambda, m)$, in the particular case of an atom exposed to a pure magnetic field $\mathbf{B}$ directed along $\left(\theta_{B}, \phi_{B}\right)$ may be written as

$$
F_{n, n^{\prime}}(\lambda, m)=F_{M_{n}, M_{n}^{\prime}}(\lambda, m)=(-1)^{M_{n}^{\prime}-m} C\left(1,1, \lambda ; M_{n},-M_{n}^{\prime}, M_{\lambda}\right) \sqrt{4 \pi}[\lambda]^{-1} Y_{\lambda M_{\lambda}}\left(\theta_{B}, \phi_{B}\right),
$$

in terms of the spherical harmonics. From Eq. (63) we can recover the Hanle-Zeeman scattering matrix of Stenflo [14] by setting the electric field strength to zero, and employing Eq. (67). The profile functions appearing in Eq. (63) are in the atomic frame. To obtain the Hanle-Zeeman scattering matrix, one needs to follow exactly the procedure outlined in [14], to transform $\phi_{n}$ to the laboratory frame through a Doppler convolution. In the weak field limit, we recover the well-known Hanle scattering phase matrix of M. Landi Degl'Innocenti and E. Landi Degl'Innocenti [38], by assuming $\phi_{M_{n}} \phi_{M_{n}^{\prime}}^{*}$ to be independent of $M_{n}, M_{n}^{\prime}$. In the strong field limit, $g B$ is large compared to the line widths. Hence the three Zeeman component lines are well separated. If we neglect the coupling between the Zeeman substates (drop the summation over $n$ in Eq. (63), by setting $n=n^{\prime}$ ), we recover the restrictive phase matrix of Obridko [39], which is basically a modified resonance scattering by individual Zeeman components.

\section{Numerical results and discussions}

The calculations presented in this paper are applicable to magnetic fields of arbitrary strength, and also the presence of quadrupole electric fields surrounding the radiating atom. To check the correctness of our derivation, we have reproduced the results of Stenflo ([14], Fig. 3) for the particular case of Hanle-Zeeman effect [40]. In weak magnetic fields, pure Hanle effect prevails. In strong fields, the Zeeman effect is the dominant process. In intermediate fields, there is a smooth transition from weak field Hanle effect to the strong field Zeeman effect. These two effects exhibit relative dominance in different regimes of field strength, but they fundamentally overlap over the entire regime.

We consider the simplest case of a $J=0 \rightarrow 1 \rightarrow 0$ type transition which produces a standard Zeeman triplet. In this section we present the results of a single scattering experiment (see Eq. (50)). We consider a $90^{\circ}$ scattering of an unpolarized beam of radiation incident on the atom. The incident radiation is also assumed to
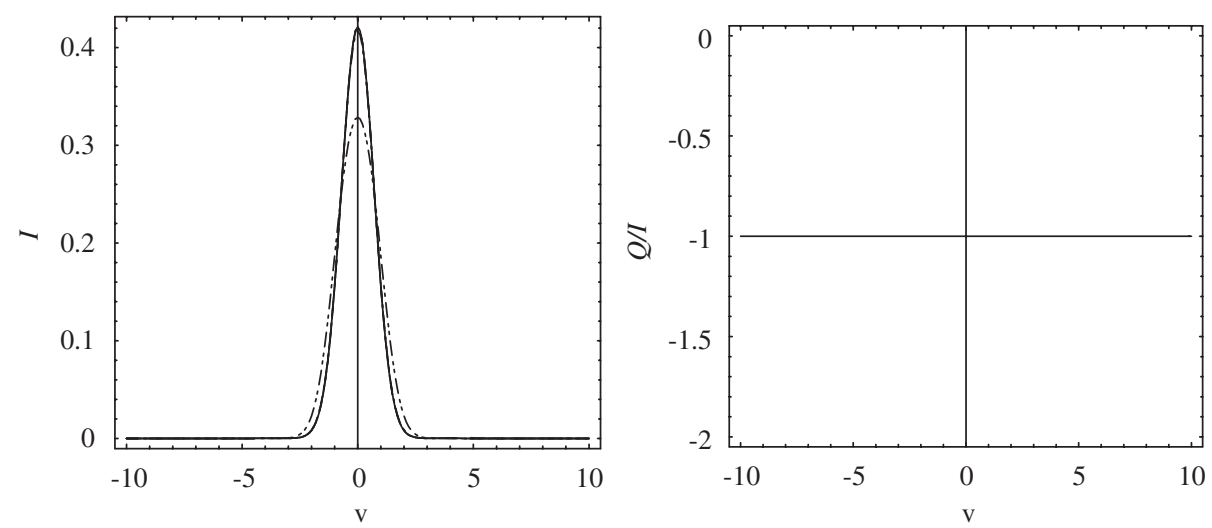

Fig. 3. Stokes profiles for pure magnetic field case. Magnetic field is oriented along the $Z$-axis of the ARF (see Fig. 1). The model parameter employed are: $a=0.004$, the scattering geometry defined by $\theta^{\prime}=0^{\circ}, \phi^{\prime}=0^{\circ} ; \theta=90^{\circ}, \phi=45^{\circ}$, and the magnetic field strength defined by $v_{B}=0.0008$ (solid line), 0.004 (dotted line), 0.02 (dashed line), 0.1 (dash-dotted line), 0.5 (dash-double dotted line) and 2.5 (dash-triple dotted line). Notice a constant degree of linear polarization $Q / I=-100 \%$. 
be frequency independent (broadband pulse). Since $\mathbf{S}^{\prime}=(1,0,0,0)^{\mathrm{T}}$, the scattered Stokes intensity components are nothing but $(I, Q, U, V)=\left(\mathscr{R}_{11}, \mathscr{R}_{21}, \mathscr{R}_{31}, \mathscr{R}_{41}\right)$, which measure the maximum degree of anisotropy for a given angle of scattering. The external magnetic field is assumed to be oriented along the $Z$-axis of the ARF (laboratory) (see Fig. 1).

The scattering is assumed to be frequency coherent in the laboratory frame. A Voigt profile function with a damping parameter $a=0.004$ in units of the Doppler width $\Delta v_{D}=\left(v_{0} / c\right) \sqrt{2 k T / M_{a}}$ is employed to compute all the results. In Section 4.1 we present the results for pure magnetic case. In Section 4.2 the results of scattering on atom immersed in pure electric quadrupole field is considered. Finally in Section 4.3 we consider the combined case of uniform magnetic and quadrupole electric fields.

\subsection{The pure magnetic field case}

Fig. 3 shows singly scattered polarization profiles for this case. The field strength $B$ is chosen to represent the entire regime of Hanle-Zeeman effect $\left(v_{B}=0.0008-2.5\right.$ in steps of a factor 5). The splitting parameter $v_{B}$ is defined as $v_{B}=v_{L} / \Delta v_{D}$ where $v_{L}=e B / 4 \pi m c$ is the Larmor frequency. The geometry of scattering chosen by us $\left(\theta^{\prime}=0^{\circ}, \phi^{\prime}=0^{\circ} ; \theta=90^{\circ}, \phi=45^{\circ}\right)$ corresponds to Stokes $I$ and $Q$ given by

$$
I=\frac{3}{8}\left[\phi_{1} \phi_{1}^{*}+\phi_{-1} \phi_{-1}^{*}\right] ; \quad Q=-I .
$$

The profile functions $\phi_{1}$ and $\phi_{-1}$, are now in the laboratory frame. They are obtained by a convolution of the atomic frame Lorentzian (see Eq. (22)) with the Doppler profile. The real part of $\phi_{1}$ for example, is a Voigt function whereas the imaginary part is a Faraday-Voigt function (see [14]). In the laboratory frame the frequency is expressed in dimensionless units $\left(v=\left(v_{0}-v\right) / \Delta v_{D}\right)$. Clearly, for weak fields the Zeeman splitting is not complete. Hence the $I$ profile simply broadens without exhibiting a separation of the components. This kind of intensity profiles are very typical of Hanle and Hanle-Zeeman regime in the second solar spectrum of the Sun (see [12]). The case of $v_{B}=2.5$ represents a strong field Zeeman effect, and we clearly see a wellseparated doublet. Since the line of sight (the scattered ray) is perpendicular to the magnetic field, according to conventional Zeeman effect theory (Zeeman effect treated as absorption/emission), one expects a triplet pattern in $I$ profile, and a $Q$ profile with $\pi$ component having opposite polarization compared to the two $\sigma$ components, along with $U=V=0$. However, we now observe only a doublet in the $I$ profile (see Fig. 3), showing that the mechanism involved is indeed a 'scattering' process and not 'absorption followed by uncorrelated emission' process. With the help of classical theory of dipole scattering, one can argue that, for $90^{\circ}$ scattering and for a magnetic field along the $Z$-axis, the $\pi$ component is not excited at all by the incident radiation. Only the components with electric vibration perpendicular to the scattering plane (containing the incident and scattered ray) are excited, and hence the two $\sigma$ components appear in the $I$ profile.

From Eq. (68) and also from Fig. 3, we see that $Q / I=-1$, i.e., independent of frequency as well as the field strength. In other words scattered polarization is same as the well-known non-magnetic pure Rayleigh scattering polarization. This is to be expected, because in the weak field regime, the Hanle effect is absent for vertical magnetic fields, and the scattered polarization can arise only due to Rayleigh scattering process. In the strong field regime, both the sigma components have linear polarization of equal magnitude, and scatter independently (see Eq. (68)). Therefore, we obtain a maximum degree of linear polarization, namely $Q / I=-1$. We refer to this case as 'Zeeman scattering' or equivalently 'Rayleigh scattering in strong magnetic fields' (see also [11,23]).

\subsection{The case of pure electric quadrupole field}

Fig. 4 shows the singly scattered Stokes profiles in the presence of a pure electric quadrupole field surrounding the atom. The single scattering experiment is considered as in the pure magnetic field case. The electric field is characterized by two parameters, namely the electric splitting parameter $A$ which is taken in the same way as magnetic splitting parameter, namely $v_{A}=0.0008-2.5$ in steps of 5 , where $v_{A}$ is electric splitting parameter in Doppler width units. The asymmetry parameter $\eta$ is fixed at 1 . The upper $J_{n}=1$ level splits into three levels with energies $-2 A, 0$ and $+2 A$ corresponding to the eigenstates $\psi_{1}=|1,0\rangle, \psi_{2}=(|1,-1\rangle-$ $|1,1\rangle) / \sqrt{2}$ and $\psi_{3}=(|1,-1\rangle+|1,1\rangle) / \sqrt{2}$, respectively (see Fig. 2c of [24]). For the scattering geometry 

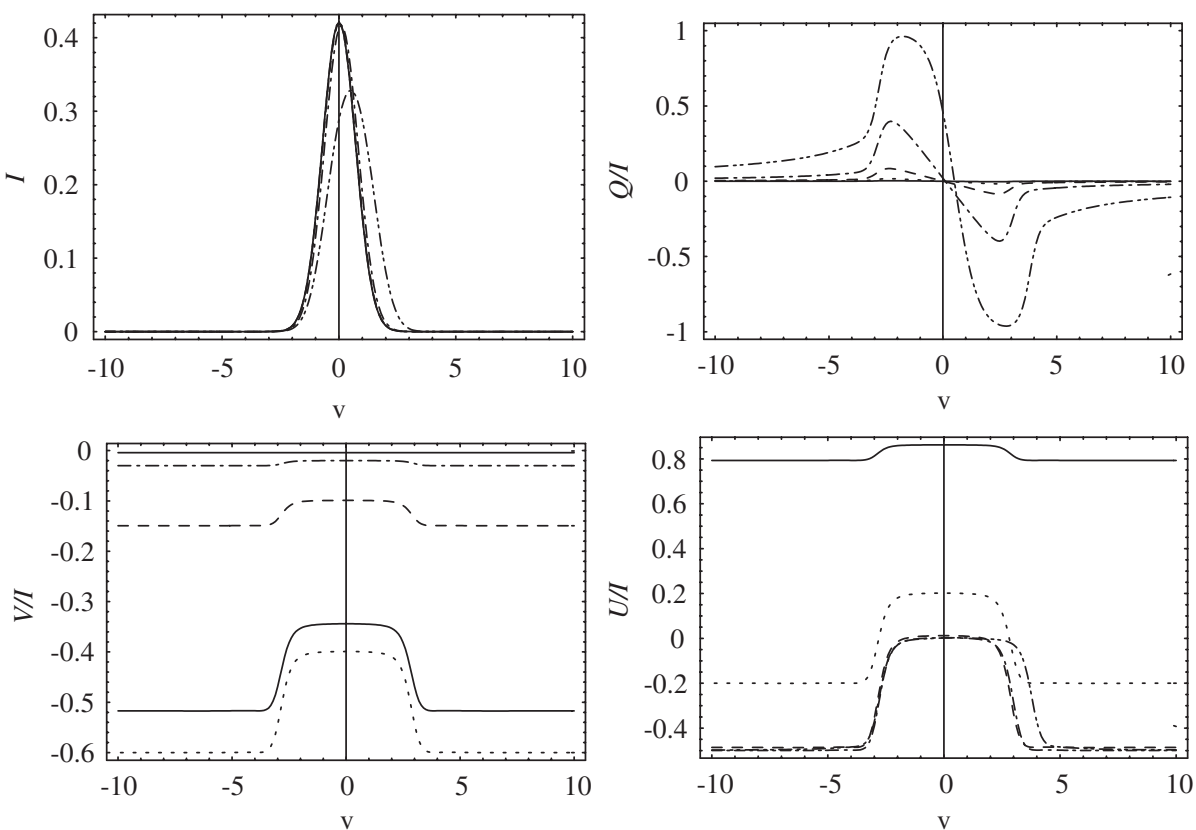

Fig. 4. Stokes line profiles for pure quadrupolar electric field case. The model parameters employed are: $a=0.004$, with the scattering geometry $\theta^{\prime}=0^{\circ}, \phi^{\prime}=0^{\circ} ; \theta=90^{\circ}, \phi=45^{\circ}$, and the electric field strength defined through the splitting parameter $v_{A}=0.0008$ (solid line), 0.004 (dotted line), 0.02 (dashed line), 0.1 (dash-dotted line), 0.5 (dash-double dotted line) and 2.5 (dash-triple dotted line) and the asymmetry parameter $\eta=1$. Notice the blue shift of the profiles compared to the pure magnetic field case for large values of $v_{A}(>0.1)$.

employed by us, the Stokes parameters are given by the analytic expressions

$$
\begin{aligned}
& I=\frac{3}{8}\left[\phi_{2} \phi_{2}^{*}+\phi_{3} \phi_{3}^{*}\right] ; \quad Q=\frac{3}{8}\left[\phi_{2} \phi_{2}^{*}-\phi_{3} \phi_{3}^{*}\right] ; \\
& U=\frac{3}{8}\left[\phi_{2} \phi_{3}^{*}+\phi_{3} \phi_{2}^{*}\right] ; \quad V=\frac{3}{8} i\left[\phi_{2} \phi_{3}^{*}-\phi_{3} \phi_{2}^{*}\right] .
\end{aligned}
$$

The profile functions $\phi_{2}$ and $\phi_{3}$ correspond to the eigenstates $\psi_{2}$ and $\psi_{3}$, respectively, and are given by

$$
\phi_{2,3}=H\left(v+(1 \mp \eta) v_{A}, a\right)+2 i F\left(v+(1 \mp \eta) v_{A}, a\right),
$$

in the laboratory frame. Here $H$ and $F$ are the well-known Voigt and Faraday-Voigt functions [14]. From Eq. (69), we note that $U$ and $V$ are generated purely due to the coupling between the eigenstates $\psi_{2}$ and $\psi_{3}$.

For weak electric fields $\left(v_{A}<0.1\right)$ the shapes of the $I$ profile are not affected significantly, when compared to the corresponding pure magnetic case (see Fig. 3). For $v_{A}>0.1$ there is a blue shift compared to the pure magnetic case, which increases gradually for larger values of $v_{A}$ until we get a doublet that is asymmetrically placed about the line center. The $Q / I$ profiles have an interesting shape, in the sense that they are similar to the $V / I$ profiles of the pure magnetic field case. For $v_{A}=0.0008$ and $0.004, Q / I$ is extremely small. As $v_{A}$ increases, $Q / I$ gradually increases, and the zero cross-over point of $Q / I$ shifts toward the blue (see for e.g. dash-triple dotted line in Fig. 4). $U / I$ and $V / I$ show interesting behavior. Unlike $Q / I$, the ratio $U / I$ takes largest value for $v_{A}=0.0008$, and is entirely positive (see upper most solid line in $U / I$ panel of Fig. 4). For $v_{A}=0.004$ (dotted line), $U / I$ takes both positive and negative values. As $v_{A}$ increases we note that $U / I$ goes to zero in the line core (except for $v_{A}=2.5$-dash-triple dotted line which shows a small positive peak around $v=2.5$ ), and becomes entirely negative and nearly constant in the wings. The shape of $V / I$ is similar to that of $U / I$ (namely, taking constant values at line core and line wings with a swift transition around $v \simeq 3$ ). The magnitude of $V / I$ initially increases with $v_{A}$ and then rapidly decreases toward zero, as $v_{A}$ increases. For $v_{A}>0.1$, the $V / I$ is nearly zero throughout the line profile (see Fig. 4).

If the mechanism involved is a pure emission process, then one would expect all the three wave functions $\psi_{1,2,3}$ to contribute and produce line components in Stokes $I$, along with $Q=V=0$ and $U \neq 0$ (see Eqs. (69)-(72) in [24]). However, when the interaction of radiation is treated as scattering (represented through 
angular correlations between incident and scattered ray), we see that only a doublet is seen in Stokes $I$ (since $\psi_{1}$ is not excited at all according to Eq. (69)) with $Q, U$ and $V$ non-zero. This is the essential difference between the spontaneous emission process and the scattering process in quadrupole electric fields. We have also computed the Stokes profiles for the $\eta=0.5$ case, and find that they do not differ qualitatively from $\eta=1$ case, except for changes caused by different amount of level splitting.

\subsection{The case of combined magnetic and quadrupole electric fields}

In Fig. 5 we show the Stokes profiles for this case. We employ ratio $A / B=0.5$ (which defines the relative strength of electric field with respect to the magnetic field), and the asymmetry parameter $\eta=1$. The splitting parameter $v_{B}(=0.0008-2.5)$ is employed as in the pure magnetic case. The simplest geometry of the combined magnetic and quadrupole fields with $\mathbf{B}$ along the $Z$-axis of the PAF, and PAF itself coinciding with the ARF, is employed in the computation of the results in this section. The scattering geometry is same as in Figs. 3 and 4. The upper level $J_{\mathrm{u}}=1$ is split into three levels $n=1-3$ with energies

$$
E_{1}=-2 r B ; \quad E_{2,3}=\left[r \mp\left(r^{2} \eta^{2}+1\right)^{1 / 2}\right] B,
$$

where $r=A / B$. The corresponding eigenstates are given by (see [24])

$$
\psi_{1}=|1,0\rangle ; \quad \psi_{2}=b_{1}|1,-1\rangle+b_{2}|1,1\rangle ; \quad \psi_{3}=-b_{2}|1,-1\rangle+b_{1}|1,1\rangle,
$$

where the interference coefficients are defined by

$$
\begin{aligned}
& b_{1}=\frac{r \eta+1+\left(r^{2} \eta^{2}+1\right)^{1 / 2}}{2\left[r^{2} \eta^{2}+1+r \eta\left(r^{2} \eta^{2}+1\right)^{1 / 2}\right]^{1 / 2}} ; \\
& b_{2}=\frac{r \eta-1+\left(r^{2} \eta^{2}+1\right)^{1 / 2}}{2\left[r^{2} \eta^{2}+1+r \eta\left(r^{2} \eta^{2}+1\right)^{1 / 2}\right]^{1 / 2}} .
\end{aligned}
$$

Fig. 6 shows the energy level splitting for spin-1 upper level, exposed to the simultaneous presence of an external electric quadrupole and uniform magnetic fields. Notice that 'level-crossing' occurs when the electric
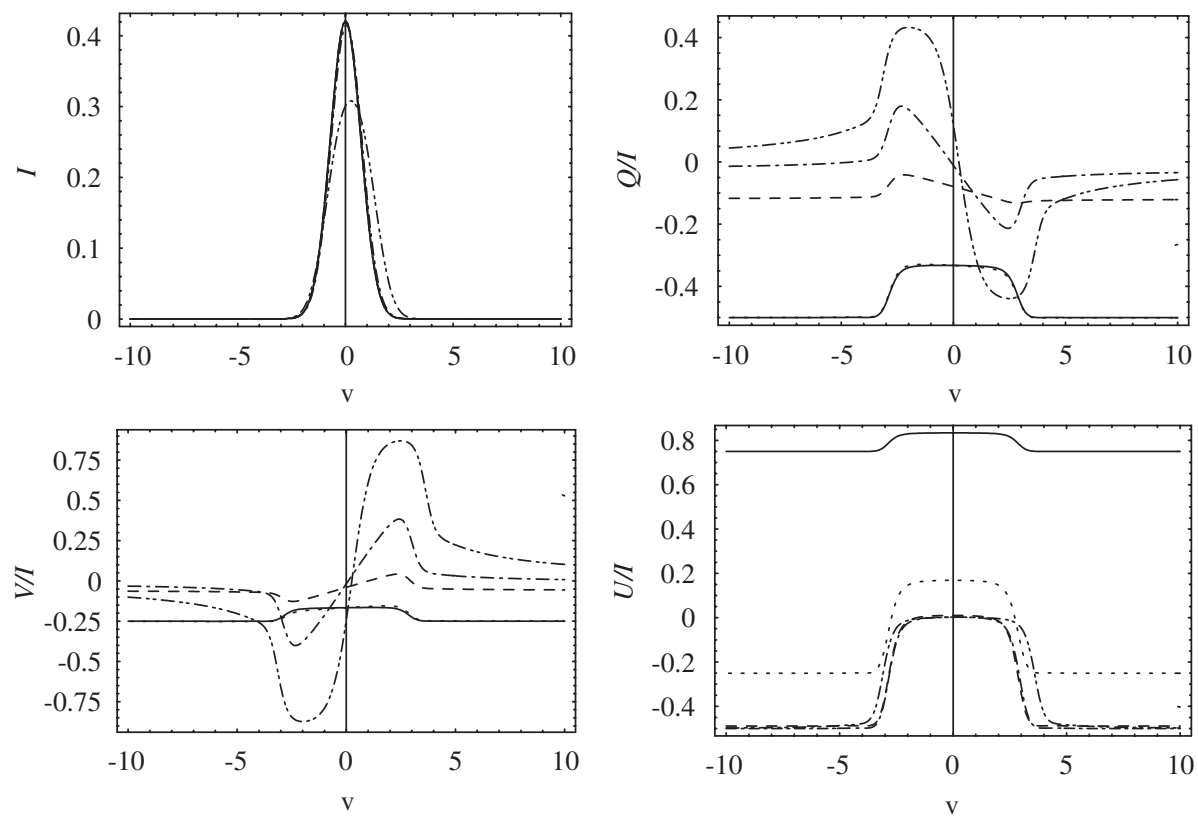

Fig. 5. Stokes profiles for combined quadrupolar electric and uniform magnetic field case. The ratio $r=A / B=0.5$ and the asymmetry parameter $\eta=1$. Other input model parameters, scattering geometry and the line types are same as in Fig. 3. 
field strength increases. It is worth noting that these levels are not pure states, but superposed states. The cross-over occurs for only a single value of electric quadrupole field strength at which the levels become degenerate.

Fig. 5 presented in this paper can be interpreted using the panel (c) of Fig. 6. For $r=0.5$, the energy eigenstates $E_{1}$ and $E_{2}$ are below the $A=B=0$ reference line. For the geometry chosen, the state $\psi_{1}=|1,0\rangle$ is not excited, as can be seen from the following analytic expressions for the scattered Stokes parameters:

$$
\begin{aligned}
& I=\frac{3}{8}\left[\phi_{2} \phi_{2}^{*}+\phi_{3} \phi_{3}^{*}\right] ; \\
& Q=\frac{3}{8}\left[2 b_{1} b_{2}\left(\phi_{3} \phi_{3}^{*}-\phi_{2} \phi_{2}^{*}\right)+i\left(b_{1}^{2}-b_{2}^{2}\right)\left(\phi_{3} \phi_{2}^{*}-\phi_{2} \phi_{3}^{*}\right)\right] ; \\
& U=\frac{3}{8}\left[\phi_{2} \phi_{3}^{*}+\phi_{3} \phi_{2}^{*}\right] ; \\
& V=\frac{3}{8}\left[\left(b_{1}^{2}-b_{2}^{2}\right)\left(\phi_{2} \phi_{2}^{*}-\phi_{3} \phi_{3}^{*}\right)+2 i b_{1} b_{2}\left(\phi_{3} \phi_{2}^{*}-\phi_{2} \phi_{3}^{*}\right)\right] .
\end{aligned}
$$
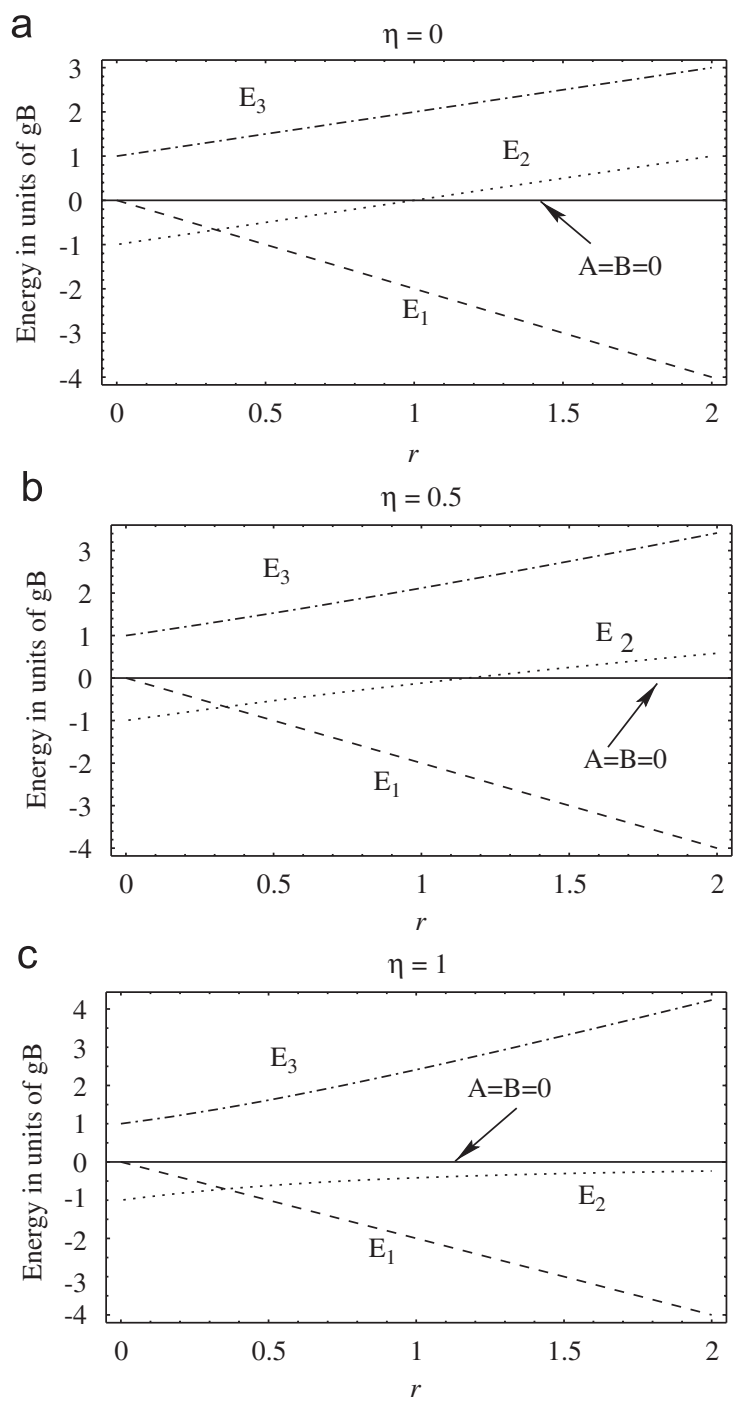

Fig. 6. Energy level diagrams showing the combined effect of magnetic and electric quadrupole fields. The panels (a-c) represent different asymmetry parameters $\eta$. The energies $E_{1}, E_{2}$ and $E_{3}$ corresponding to the states $\psi_{1}, \psi_{2}$ and $\psi_{3}$, respectively, are plotted. The value $r=0$ corresponds to the pure Zeeman case. 
The profile functions $\phi_{2}$ and $\phi_{3}$ in the above expressions correspond to the eigenstates $\psi_{2}$ and $\psi_{3}$, respectively, and are given by

$$
\phi_{2,3}=H\left(v+\left(r \mp \sqrt{r^{2} \eta^{2}+1}\right) v_{B}, a\right)+2 i F\left(v+\left(r \mp \sqrt{r^{2} \eta^{2}+1}\right) v_{B}, a\right),
$$

in the laboratory frame for any $\eta$.

The Stokes $I$ profiles in the combined case are quite similar in amplitude and shape to those in the pure magnetic case, except for the position of the component lines (for $v_{B}>0.1$ ). For $v_{B}=0.0008$ and 0.004 (solid and dotted lines which nearly overlap on each other in $Q / I$ panel of Fig. 5), the $Q / I$ profiles are very different compared to the corresponding pure electric field case, and are entirely negative. For $v_{B}>0.004$, these $Q / I$ profiles resemble their corresponding counterparts of the pure electric field case in terms of the frequency dependence. Clearly, there is a decrease in the magnitude of $Q / I$ when compared to both the pure magnetic and the pure electric case.

$U / I$ in the combined case is quite similar to the pure electric case (compare $U / I$ in Eq. (69) with Eq. (74) for $r=0.5$ and $\eta=1)$. In other words, we can conclude that for this particular geometry the entire frequency dependence of the $U / I$ comes from the electric field effect. The only difference is in $v_{B}=2.5$ (dash-triple dotted line) case, wherein the small peak is enhanced as well as it is now centered around 1.5 , unlike in pure electric field case. $V / I$ profiles in combined case bear resemblance to $Q / I$ profiles in shape, differing only in magnitude and sign (for $v_{B}>0.0004$ ). Clearly, the quadrupolar electric field produces an additional depolarization in $Q / I$ (compared to the $Q / I$ of pure magnetic case in Fig. 3), and a rotation of the plane of polarization (or generation of new $U / I$ ). Therefore in the special geometry of vertical magnetic fields (B parallel to the $Z$-axis of PAF and ARF simultaneously), if one observes strong $U / I$ and $V / I$ signals as well as relatively smaller $Q / I$ signals in a $90^{\circ}$ scattering (e.g. as in the case of extreme limb observations of the solar chromosphere; or like the special scattering geometry that we considered for discussion), it could indicate the asymmetries arising from the quadrupolar electric fields surrounding the atom. Notice that in the absence of electric fields, $U / I=V / I=0$ and $Q / I=-1$ for the vertical magnetic fields (see Fig. 3). Another diagnostic indicator of quadrupolar electric fields is the net blue shift of the Stokes profiles, unlike the linear Stark effect which produces symmetric shifts with respect to the line center.

For the sake of discussion, we have computed the scattered Stokes profiles for $\eta=0$ also in the combined case. This case is interesting, because, from Fig. 6 we observe that for $r \leqslant 0.5$, the splitting is independent of $\eta$, namely the splitting pattern is same for both $\eta=0$ and 1. As a result, the Stokes $I$ for both $\eta=0$ and 1 cases are nearly identical. This can be understood from Eq. (74) by setting $\eta=0$. The profile functions $\phi_{2,3}$ are now given by Eq. (75) with $\eta=0$. We note that the real part of $\phi_{2}$ for $\eta=0$ and 1 , respectively, are $H\left(v-0.5 v_{B}, a\right)$ and $H\left(v-0.62 v_{B}, a\right)$. Similarly for $\phi_{3}$, corresponding real parts are $H\left(v+1.5 v_{B}, a\right)$ and $H\left(v+1.62 v_{B}, a\right)$. For this reason, the Stokes $I$ as well as $U / I$ profile for $\eta=0$ and 1 are nearly identical. However the $Q / I$ and $V / I$ profiles are quite different (see Eqs. (74)). The $Q / I$ profiles of $\eta=0$ case have shapes and magnitudes similar to the $V / I$ profiles of Fig. 4 . The $V / I$ profiles of $\eta=0$ case have shapes and magnitudes similar to $Q / I$ profiles of Fig. 4, except for a sign difference.

\section{Conclusions}

The scattering matrices for the combined effect of electric quadrupole field and uniform magnetic fields (of arbitrary strength) are derived using quantum electrodynamic approach. The scattering matrix for Hanle-Zeeman effect is validated by comparing with the published results of Stenflo [14]. The quadrupole electric field is characterized by strength and asymmetry parameters, which produce unique diagnostic signatures that may be employed to detect the electric charge distribution asymmetries in the solar atmosphere. The theoretical formulation is quite general and can handle not only the simplest case of a triplet $(J=0 \rightarrow 1 \rightarrow 0)$ transition that is employed for illustrations in this paper, but also an arbitrary choice of quantum numbers. We have demonstrated the properties of the coherence or interference phenomena, like strong field Zeeman scattering, Hanle and magnetic Raman scattering that are important in the interpretation of spectral lines in the second solar spectrum. 


\section{Acknowledgments}

GR is grateful to Professors B.V. Sreekantan, R. Cowsik, J.H. Sastry, R. Srinivasan and S.S. Hasan for facilities provided for research at the Indian Institute of Astrophysics. Y.Y. Oo wishes to thank the Director of Indian Institute of Astrophysics, the Chairman, Physics Department, Bangalore University for extending the research facilities. She also acknowledges ICCR, Government of India for financial support to visit India, during which the major part of the work was carried out. MS is financially supported by Council of Scientific and Industrial Research (CSIR), through a SRF (Grant No: 9/890(01)/2004-EMR-I), which is gratefully acknowledged. Authors would like to thank Mr. K. Nagaraju for useful discussions.

\section{References}

[1] Hale GE. On the probable existence of a magnetic field in sun-spots. Astrophys J 1908;28:315-43.

[2] Chandrasekhar S. Radiative transfer. Oxford: Clarendon Press; 1950.

[3] Landi Degl'Innocenti E. Polarization in spectral lines. I - a unifying theoretical approach. Sol Phys 1983;85:3-31.

[4] Landi Degl'Innocenti E. Polarization in spectral lines. II - a classification scheme for solar observations. Sol Phys 1983;85:33-40.

[5] Landi Degl'Innocenti E. Polarization in spectral lines. III - resonance polarization in the non-magnetic, collisionless regime. Sol Phys 1984;91:1-26.

[6] Landi Degl'Innocenti E. Polarization in spectral lines. IV-resonance polarization in the Hanle effect, collisionless regime. Sol Phys 1985;102:1-20.

[7] Landi Degl'Innocenti E, Bommier V, Sahal-Bréshot S. Resonance line polarization and the Hanle effect in optically thick media. I-formulation for the two-level atom. Astron Astrophys 1990;235:459-71.

[8] Landi Degl'Innocenti E, Bommier V, Sahal-Bréchot S. Resonance line polarization for arbitrary magnetic fields in optically thick media. I. Basic formalism for a 3-dimensional medium. Astron Astrophys 1991;244:391-400.

[9] Landi Degl'Innocenti E, Bommier V, Sahal-Bréchot S. Resonance line polarization for arbitrary magnetic fields in optically thick media. II. Case of a plane-parallel atmosphere and absence of Zeeman coherences. Astron Astrophys 1991;244:401-8.

[10] Bommier V. Master equation theory applied to the redistribution of polarized radiation, in the weak radiation field limit. I. Zero magnetic field case. Astron Astrophys 1997;328:706-25.

[11] Bommier V. Master equation theory applied to the redistribution of polarized radiation in the weak radiation field limit. II. Arbitrary magnetic field case. Astron Astrophys 1997;328:726-51.

[12] Stenflo JO. Solar magnetic fields - polarized radiation diagnostics. Dordrecht: Kluwer Academic Publishers; 1994.

[13] Stenflo JO. Quantum interferences, hyperfine structure, and Raman scattering on the Sun. Astron Astrophys 1997;324:344-56.

[14] Stenflo JO. Hanle-Zeeman scattering matrix. Astron Astrophys 1998;338:301-10.

[15] Bommier V, Stenflo JO. Partial frequency redistribution with Hanle and Zeeman effects. Non-perturbative classical theory. Astron Astrophys 1999;350:327-33.

[16] Favati B, Landi Degl'Innocenti E, Landolfi M. Resonance scattering of Lyman-alpha in the presence of an electrostatic field. Astron Astrophys 1987;179:329-38.

[17] Casini R, Landi Degl'Innocenti E. The polarized spectrum of hydrogen in the presence of electric and magnetic fields. Astron Astrophys 1993;276:289-302.

[18] Casini R. Resonance scattering formalism for the hydrogen lines in the presence of magnetic and electric fields. Phys Rev A 2005;71:062505-1-17.

[19] Brillant S, Mathys G, Stehle C. Hydrogen line formation in dense magnetized plasmas. Astron Astrophys 1998;339:286-97.

[20] Trujillo Bueno J, Moreno Insertis F, Sánchez F, editors. Astrophysical spectropolarimetry. Cambridge: Cambridge University Press; 2002.

[21] Landi Degl'Innocenti E, Landolfi M. Polarization in spectral lines. Dordrecht: Kluwer Academic Publishers; 2004.

[22] Sampoorna M, Nagendra KN, Stenflo JO. Hanle-Zeeman redistribution matrix I. Classical theory expressions in the laboratory frame. Astrophys J 2007 (in press).

[23] Sampoorna M, Nagendra KN, Stenflo JO. Hanle-Zeeman redistribution matrix II. Comparison of classical and quantum electrodynamic approaches. Astrophys J 2006 (submitted for publication).

[24] Oo YY, Nagendra KN, Ananthamurthy S, Vijayashankar R, Ramachandran G. Polarization of line radiation in the presence of external electric quadrupole and uniform magnetic fields. JQSRT 2004;84:35-64.

[25] Oo YY, Nagendra KN, Ananthamurthy S, Sirsi S, Vijayashankar R, Ramachandran G. Polarization of line radiation in the presence of external electric quadrupole and uniform magnetic fields: II. Arbitrary orientation of magnetic field. JQSRT 2005;90:343-66.

[26] Rose ME. Elementary theory of angular momentum. New York: John Wiley; 1957.

[27] Hanle W. Über magnetische Beeinflussung der Polarisation der Resonanz fluoreszenz. Z Phys 1924;30:93-105.

[28] Bransden BH, Joachain CJ. Physics of atoms and molecules. London: Longman; 1993.

[29] Shore BW, Menzel DH. Principles of atomic spectra. New York: Wiley; 1968.

[30] Muha GM. Exact solution of the NQR $I=1$ eigenvalue problem for an arbitrary asymmetry parameter and Zeeman field strength and orientation. J Chem Phys 1980;73:4139-40. 
[31] Muha GM. Exact solution of the eigenvalue problem for a spin-3/2 system in the presence of a magnetic field. J Magn Res 1983;53:85-102.

[32] Jauch JM, Rohrlich F. The theory of photons and electrons. New York: Addison-Wesley; 1955.

[33] Hamilton J. The theory of elementary particles. Oxford; 1959.

[34] Merzbacher E. Quantum mechanics. New York: Wiley; 1970.

[35] Weisskopf V, Wigner E. Berechnung der natürlichen Linienbreite auf Grund der Diracschen Lichttheorie. Z Phys 1930;63:54-73.

[36] Weisskopf V, Wigner E. Über die natürliche Linienbreite in der Strahlung des harmonischen Oszillators. Z Phys 1930;65:18-29.

[37] McMaster WH. Matrix representation of polarization. Rev Mod Phys 1961;33:8-27.

[38] Landi Degl'Innocenti M, Landi Degl'Innocenti E. An analytical expression for the Hanle-effect scattering phase matrix. Astron Astrophys 1988;192:374-8.

[39] Obridko VN. Scattering matrix for radiation in a magnetic field. Sov Phys Astron 1965;19:77-80.

[40] Oo YY. Studies in astrophysical line formation theory. PhD thesis; 2004. 